\title{
Space Launch System Booster Separation Supersonic Powered Testing with Surface and Off-body Measurements
}

\author{
Courtney S. Winski*, Paul M. Danehy ${ }^{\dagger}$ A. Neal Watkins ${ }^{\ddagger}$, Patrick R. Shea ${ }^{\S}$ \\ NASA Langley Research Center, Hampton, VA 23681, USA \\ Jamie G. Meeroff ${ }^{\text {II }}$ \\ Science and Technology Corp., Moffett Field, CA 94035, USA \\ K. Todd Lowe" \\ Virginia Polytechnic Institute and State University, Blacksburg, VA 24061, USA
}

Heather P. Houlden**

ViGYAN, Hampton, VA 23666, USA

\begin{abstract}
A wind tunnel test was run in the NASA Langley Unitary Plan Wind Tunnel simulating the separation of the two solid rocket boosters (SRB) from the core stage of the NASA Space Launch System (SLS). The test was run on a $0.9 \%$ scale model of the SLS Block 1B Cargo (27005) configuration and the SLS Block 1B Crew (28005) configuration at a Mach of 4.0. High pressure air was used to simulate plumes from the booster separation motors located at the nose and aft skirt of the two boosters. Force and moment data were taken on both SRBs and on the core stage. Schlieren still photos and video were recorded throughout testing. A set of points were acquired using Cross-correlation Doppler Global Velocimetry (CCDGV) readings to get 3 component velocity measurements between the core and the left-hand SRB. The CCDGV laser was utilized to record flow visualization in the same location, between the core and the left-hand SRB. Pressure Sensitive Paint data were taken on a separate set of runs. Computational Fluid Dynamics (CFD) runs were computed on a subset of the wind tunnel data points for comparison. A combination of the force/moment, CCDGV and Pressure Sensitive Paint (PSP) data (as well as schlieren images) at the CFD-specified test conditions will be used to validate the CFD simulations that will be used to build an SLS booster separation database at flight conditions.
\end{abstract}

Notice to the Reader - The Space Launch System, including its predicted performance and certain other features and characteristics, has been defined by the U.S. Government to be Sensitive But Unclassified (SBU). Information deemed to be SBU requires special protection and may not be disclosed to an international audience. To comply with SBU restrictions, details such as absolute values have been removed from some plots and figures in this paper. It is the opinion of the authors that despite these alterations, there is no loss of meaningful technical content.

\section{Nomenclature}

$C_{p} \quad=\quad$ Coefficient of pressure

$C_{T} \quad=$ SRB thrust coefficient

delxlh = Left-hand SRB axial translation [in.]

delxrh $=$ Right-hand SRB axial translation [in.]

delylh = Left-hand SRB lateral translation [in.]

\footnotetext{
*Aerospace Engineer, Configuration Aerodynamics Branch, Mail Stop 499, AIAA Senior Member.

†Senior Technologist, Advanced Measurement and Data Systems Branch, Mail Stop 493, AIAA Associate Fellow.

$\doteqdot$ Research Scientist, Advanced Measurement and Data Systems Branch, Mail Stop 493, AIAA Senior Member.

$\S$ Aerospace Engineer, Configuration Aerodynamics Branch, Mail Stop 499, AIAA Member.

"Research Scientist/Engineer, Computational Aerosciences Branch, AIAA Member.

" Associate Professor, Department of Aerospace and Ocean Engineering, AIAA Associate Fellow.

** Senior Aerospace Engineer, 30 Research Drive, AIAA Senior Member.
} 


$\begin{array}{ll}\text { delyrh } & =\text { Right-hand SRB lateral translation [in.] } \\ \text { delzlh } & =\text { Left-hand SRB vertical translation [in.] } \\ \text { delzrh } & =\text { Right-hand SRB vertical translation [in.] } \\ d x & =\text { SRB axial translation [in.] } \\ d y & =\text { SRB lateral translation [in.] } \\ d z & =\text { SRB vertical translation [in.] } \\ d \psi & =\text { SRB yaw angle }\left[{ }^{\circ}\right] \\ d \theta & =\text { SRB pitch angle }\left[{ }^{\circ}\right] \\ \mathrm{HO} & =\text { Freestream stagnation pressure [psfa] } \\ M & =\text { Flight Mach number } \\ P_{0} & =\text { Total pressure [psfa] } \\ P_{\infty} & =\text { Freestream static pressure [psfa] } \\ \psi_{C R H} & =\text { Right-hand SRB yaw angle }\left[{ }^{\circ}\right] \\ Q & =\text { Dynamic pressure [psfa] } \\ R e & =\text { unit Reynolds number, per foot } \\ T_{0} & =\text { Total Temperature }\left[{ }^{\circ} \mathrm{F}\right] \\ \theta_{C R H} & =\text { Right-hand SRB pitch angle }\left[{ }^{\circ}\right] \\ u & =\text { Velocity vector component in the } X \text { direction } \\ v & =\text { Velocity vector component in the } Y \text { direction } \\ U_{\infty} & =\text { Freestream velocity [ft/s] } \\ w & =\text { Velocity vector component in the } Z \text { direction }\end{array}$

Acronyms

$\begin{array}{ll}\text { BSM } & =\text { Booster Separation Motor } \\ \text { CCDGV } & =\text { Cross-correlation Doppler Global Velocemetry } \\ \text { CFD } & =\text { Computational Fluid Dynamics } \\ \text { LSRB } & =\text { Left-hand Solid Rocket Booster } \\ \text { NASA } & =\text { National Aeronautics and Space Administration } \\ \text { PSP } & =\text { Pressure Sensitive Paint } \\ \text { RSRB } & =\text { Right-hand Solid Rocket Booster } \\ \text { SLS } & =\text { Space Launch System } \\ \text { SRB } & =\text { Solid Rocket Booster } \\ \text { UPWT } & =\text { Unitary Plan Wind Tunnel }\end{array}$

\section{Introduction}

The Space Launch System[1] (SLS) is the National Aeronautics and Space Administration's (NASA) future heavy lift launch vehicle that will be used to take crew and cargo beyond low Earth orbit (LEO). At launch, the main body of the SLS is made up of the core stage and two solid rocket boosters (SRB). The SRBs contain solid fuel that is used to provide high thrust to the vehicle at liftoff and through the portion of ascent where aerodynamic forces on the vehicle are greatest. Once the solid fuel is spent (about 2 minutes into flight), the boosters separate from the core stage and fall back to Earth. Booster separation motors (BSM), located on the nose and on the aft skirt of both SRBs, are fired for one second to push the boosters away from the core stage during separation to assure there is no recontact.

A wind tunnel test was run at the NASA Langley Unitary Plan Wind Tunnel (UPWT) to look at the aerodynamics of the booster separation event to ensure that the SRBs separate safely from the core stage during flight. The test was run at a Mach of 4.0 and at a unit Reynolds number of 1.25 million per foot. The SRBs were set at pitch and yaw angles relative to the core, and the SRB noses were set at X, Y, and Z locations relative to the core nose. BSM plumes were simulated with cold gas nozzles using high pressure air. Data were taken continuously while the SRBs were moved in the $\mathrm{X}$ direction, relative to the core. This test builds on the knowledge and experience of the SLS Block 1 (10005) booster separation test run in the same tunnel, which is described in detail by Wilcox[2].

Two core configurations were tested during this wind tunnel entry, the SLS Block 1B Cargo (27005), and the SLS Block 1B Crew (28005). The boosters and the core stage remained the same for both configurations, and the upper stage 
of the centerbody was the only hardware on the model that was changed out. Figure 1 shows the vehicle evolution of the SLS. Both boosters and the core had force and moment balances for measuring aerodynamic forces on each model component separately. Schlieren video and stills were taken throughout the test. Cross-correlation Doppler Global Velocimetry (CCDGV) and Pressure Sensitive Paint (PSP) data were taken on both model configurations. Flow visualization runs were done using the CCDGV laser allowing researchers to see some 2-D flow interactions between the core stage and the left-hand SRB.

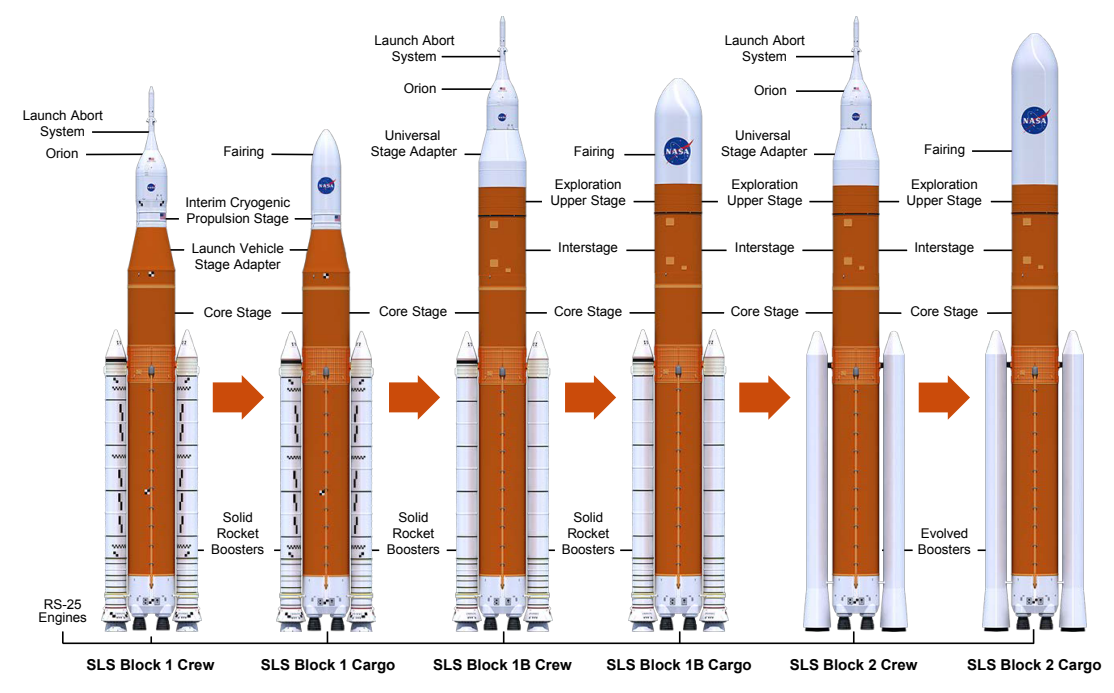

Fig. 1 Planned configurations for the SLS[1].

Wind tunnel testing with all of the core and SRB engine plumes was not possible, therefore, experimental simulations of the BSM exhaust plumes were conducted to obtain their effect on the flowfield as well as any plume interactions on the core, SRBs, and associated shocks. The measurements taken during the test will be used to verify the computational fluid dynamics (CFD) codes [3, 4] that are required to build the booster separation database [5] because it is the only method available to simulate all plumes and their interactions. The separation database will be used by analysts modeling the vehicle, specifically the Guidance, Navigation \& Control team, who will be simulating the booster separation trajectory. Information on past database development for SLS booster separation can be found in the works by Chan et al. [6], Dalle et al. [7] and Pamadi et al. [8]. The multiple flow visualization techniques used during this test will provide further understanding of the complex flow fields, and allow for another comparison to CFD.

\section{Wind Tunnel Test Description}

\section{A. NASA Langley Unitary Plan Wind Tunnel}

The NASA Langley UPWT is a closed circuit, continuous flow, variable pressure, supersonic wind tunnel. There are two test sections, a low speed (Mach 1.5 to 2.9) leg, and a high speed (Mach 2.3 to 4.6) leg. The test sections are approximately 4 feet, by 4 feet, by 7 feet long. The nozzle ahead of each test section consists of an asymmetric sliding block that allows for a continuous variation of Mach number. Six compressors are used in different combinations, or modes, along with a 100,000 horse power motor to run the tunnel. A drawing of the UPWT layout is shown in Figure 2 , and a detailed description of the tunnel can be found in Reference [9] along with calibration information. The SLS Booster Separation test was conducted in the high-speed leg of the tunnel. Test conditions are shown in Table 1.

Table 1 Test Conditions.

\begin{tabular}{cccccc}
\hline Mach & $\begin{array}{c}\operatorname{Re} \times 10^{6} \\
\text { per foot }\end{array}$ & $\begin{array}{c}P_{0} \\
\text { psfa }\end{array}$ & $\begin{array}{c}T_{0} \\
{ }^{\circ} \mathrm{F}\end{array}$ & $\begin{array}{c}\mathrm{Q} \\
\mathrm{psfa}\end{array}$ & $\begin{array}{c}P_{\infty} \\
\text { psfa }\end{array}$ \\
\hline 4.0 & 1.25 & 2311 & 150 & 170 & 15.2 \\
\hline
\end{tabular}




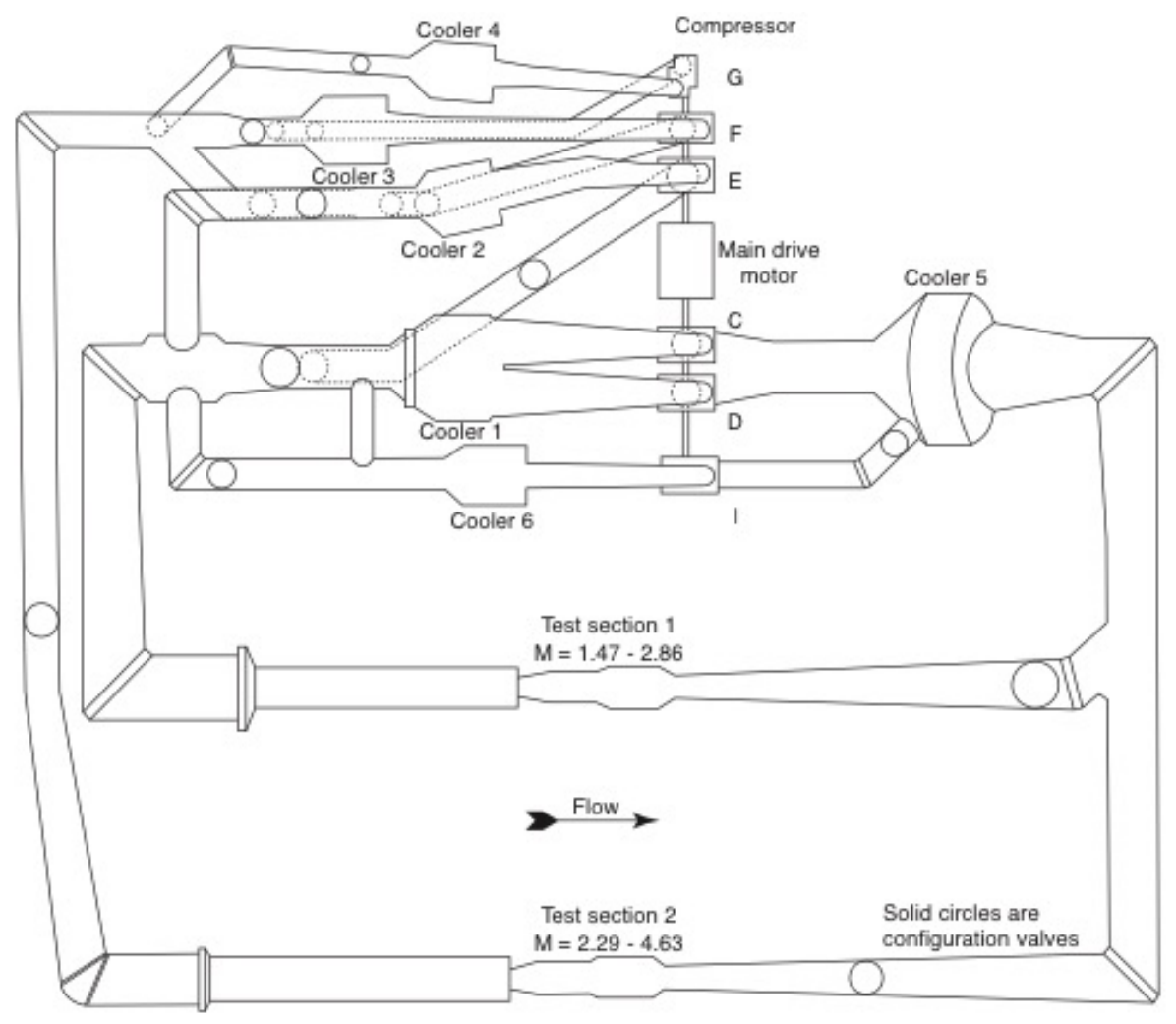

Fig. 2 Layout of the NASA Langley Unitary Plan Wind Tunnel[9].

The UPWT high pressure air system was used to supply high pressure air to both SRBs to simulate plumes from the BSMs located on the nose and aft skirt of each of the SRBs. High pressure air was continuously supplied to the 5000 psig UPWT bottle field from a central compressor station and bottle field located at the Langley Research Center. Flexible hoses connected the high pressure air system to the aft end of the SRB stings. From the stings, the high pressure air flowed into the SRB models where there were channels routing the air to forward and aft BSMs. A heater was used within the high pressure air system to heat the air to $150^{\circ} \mathrm{F}$. High pressure air was run through the entire system, including through the model and out the BSMs, with the tunnel off prior to daily testing to preheat the entire system to avoid shifts in data due to temperature fluctuations.

\section{B. Model Installation}

The model consisted of three completely separate bodies: the centerbody, the right hand SRB, and the left hand SRBs. The centerbody was mounted on a sting attached to the sidewall of the test section. The SRBs were on a separation rig mounted to the tunnel strut. Figures $3 \& 4$ show the model installed in the tunnel. The model was installed at a $90^{\circ}$ roll angle to take full advantage of the tunnel strut movement capabilities. The SRB positions were set relative to the mated launch position of the SRBs to the core body, as shown in Figures 5 \& 6 The separation rig allowed the pitch angle of the SRBs relative to the core to be set manually with the tunnel off. The yaw angle of the SRBs relative to the core, the $\mathrm{Y}$ and $\mathrm{Z}$ distances, and the BSM pressures were set with the tunnel on before each run. The SRBs were moved in the $\mathrm{X}$ direction using the tunnel strut while taking continuous data. $\mathrm{Y}$ was set using the traversing mechanism on the tunnel strut. The core could be manually set to pitch angles of $0^{\circ}$, or $2^{\circ}$, but had no other movement capability. A diagram of the separation rig can be seen in Figure 7 


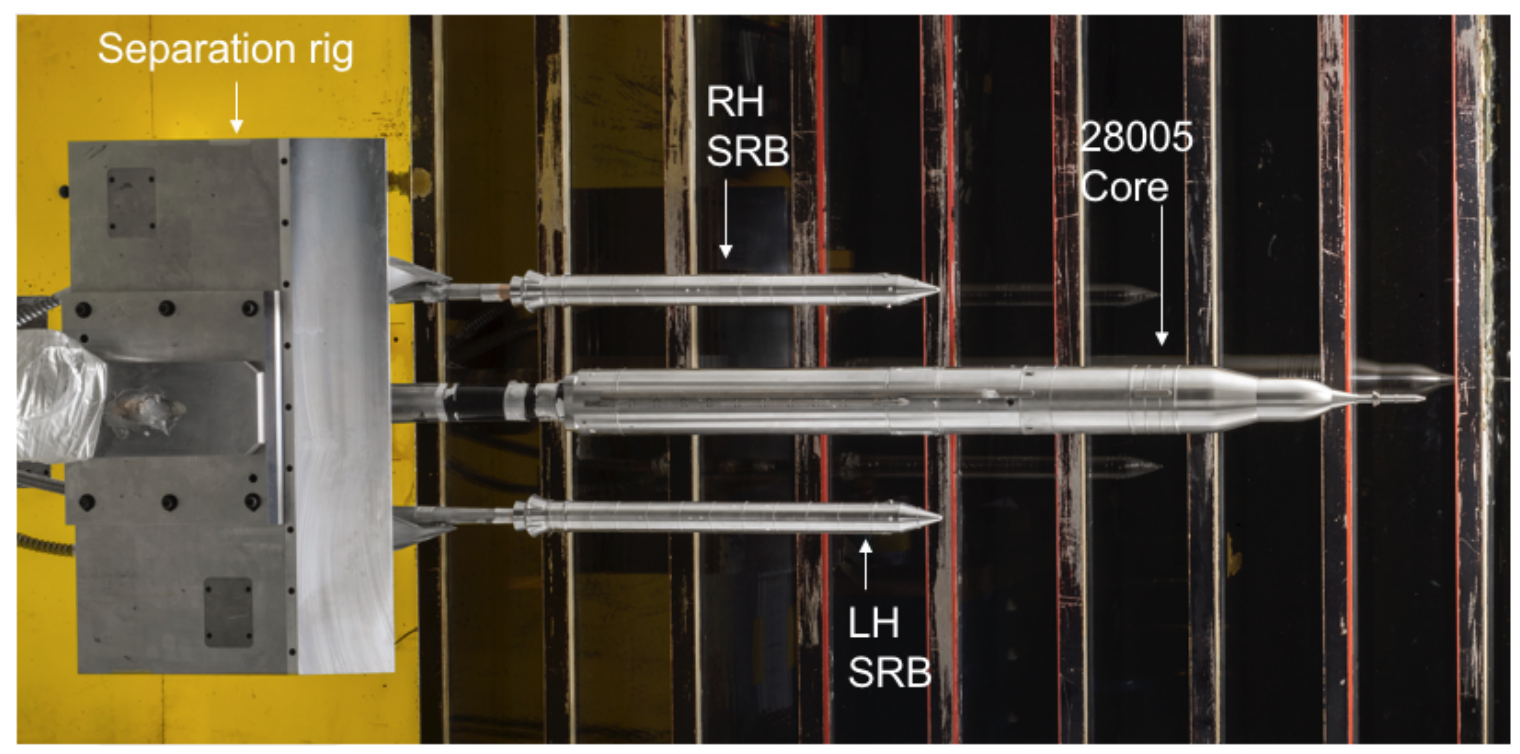

Fig. 3 SLS-28005 Configuration Installed in the UPWT.

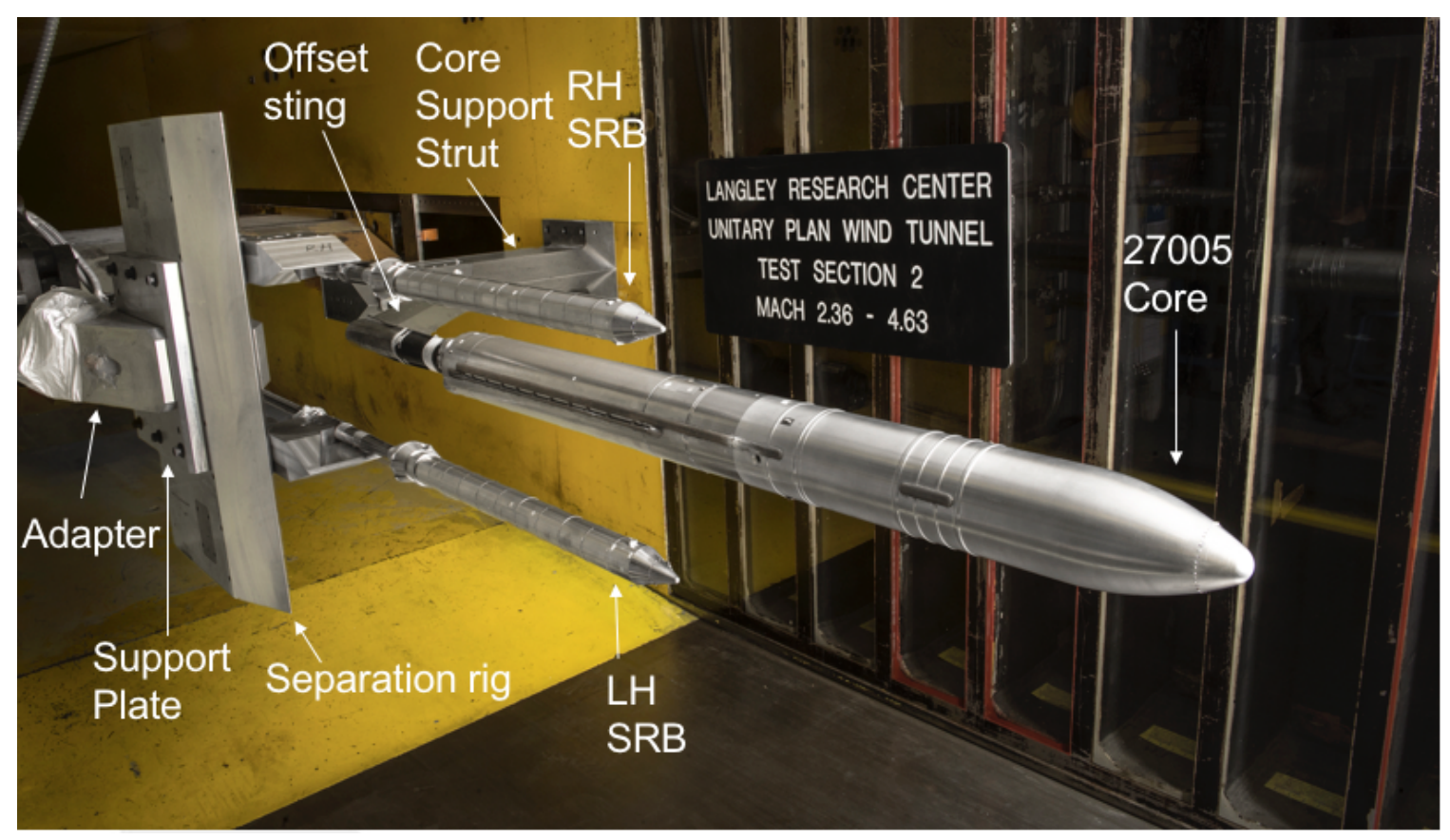

Fig. 4 SLS-27005 Configuration Installed in the UPWT.

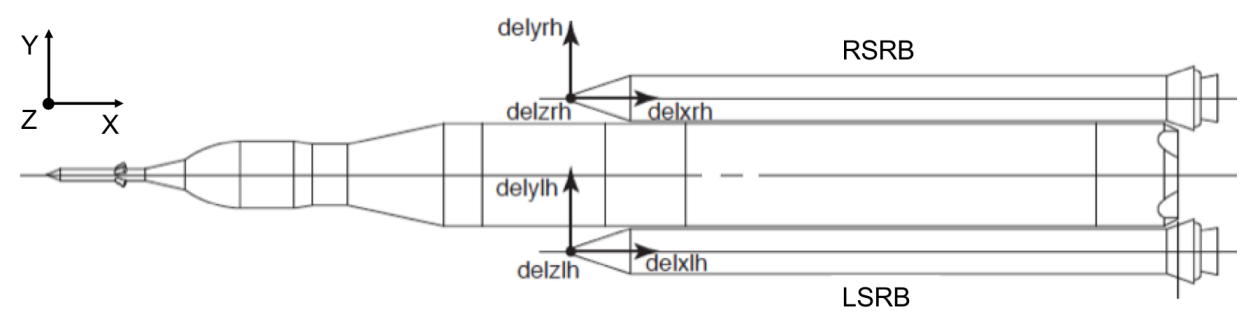

Fig. 5 Planform view of SRB delta nose position origins. 


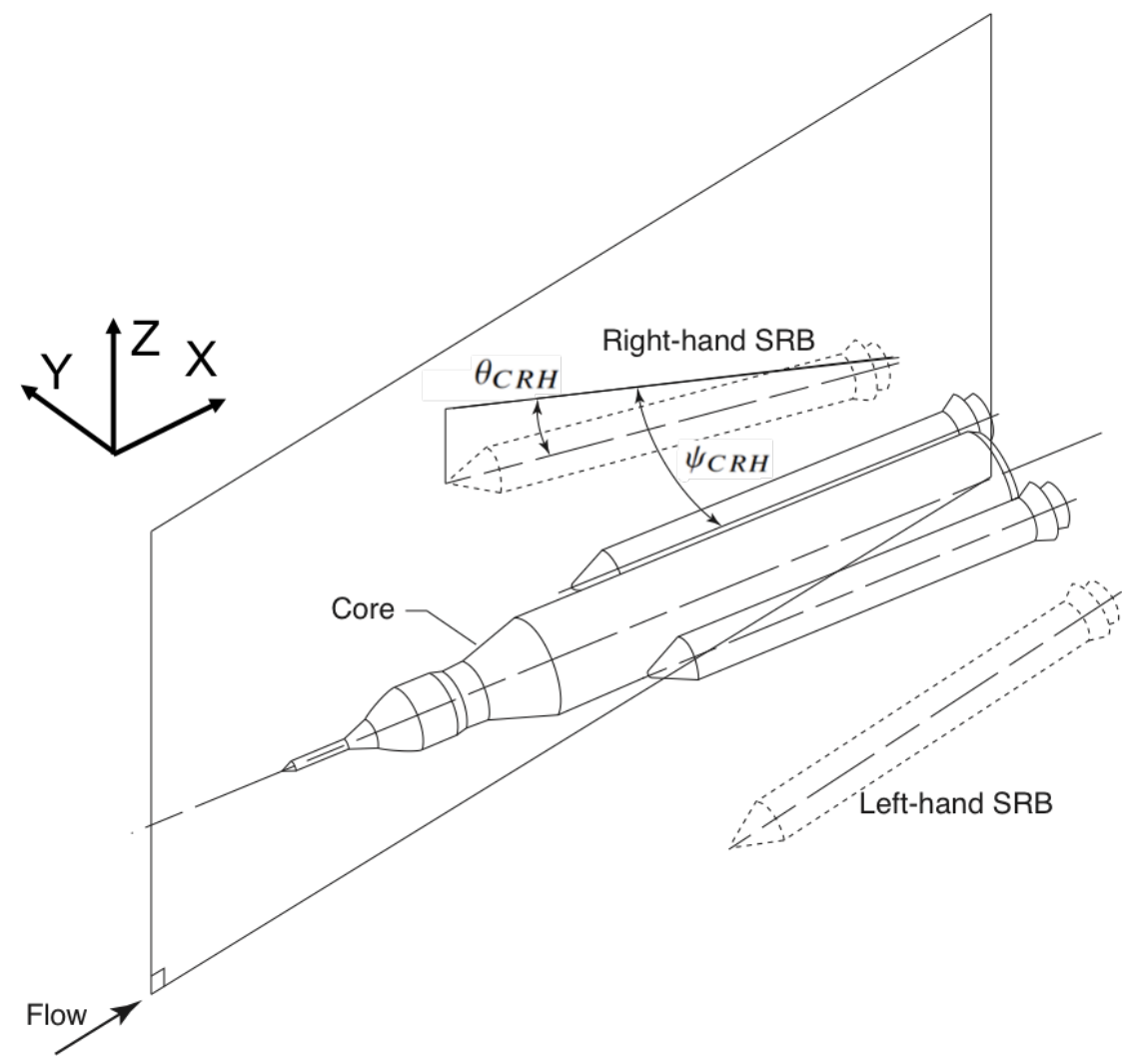

Fig. 6 Delta nose position of RH SRB from launch configuration.
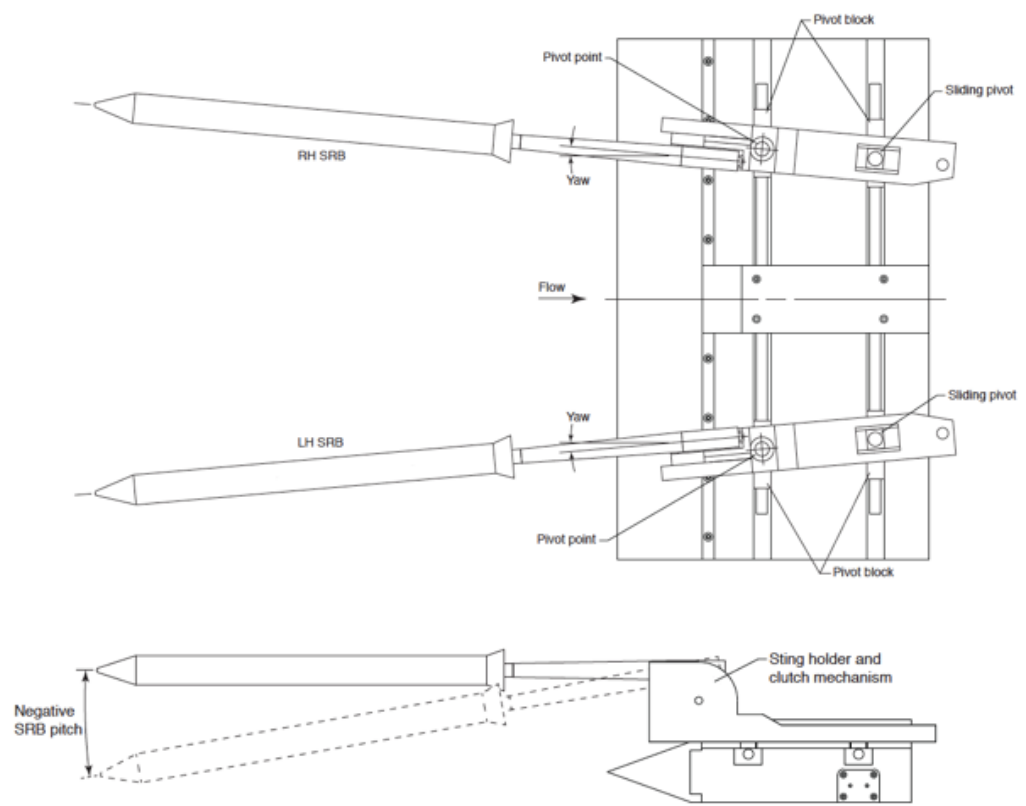

Fig. 7 Separation rig, yaw and pitch capability. 


\section{Model Description}

The test article was a $0.9 \%$ scale model of the SLS Block 1B Cargo (27005) (Figure 4), and the SLS Block 1B Crew (28005) (Figure 3) configurations. The core centerbody and the SRBs remained unchanged between the configurations while the nose of the core was switched out. The core and both SRBs were each equipped with a balance for measuring forces and moments on the vehicle during separation testing. The model included protuberances and flow-through BSMs on the nose and aft skirt of both SRBs. Figure 8 shows the forward and aft BSM nozzles. Testing was also conducted on an alternate set of aft BSMs on which three of the four nozzles were rotated $15^{\circ}$ about the BSM body axis. All of the CCDGV, flow visualization, and PSP data shown in this report were taken with the original aft BSMs installed while the schlieren images were taken with the rotated nozzles. All of the CFD was run with the rotated nozzles.

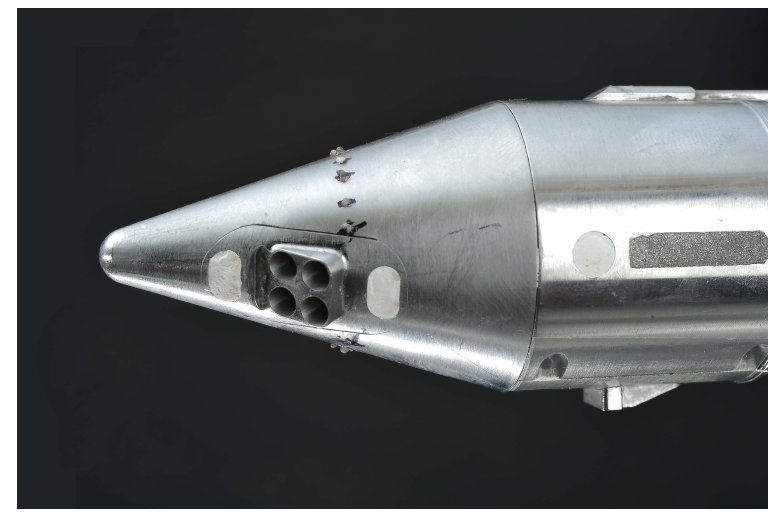

(a) Forward BSM

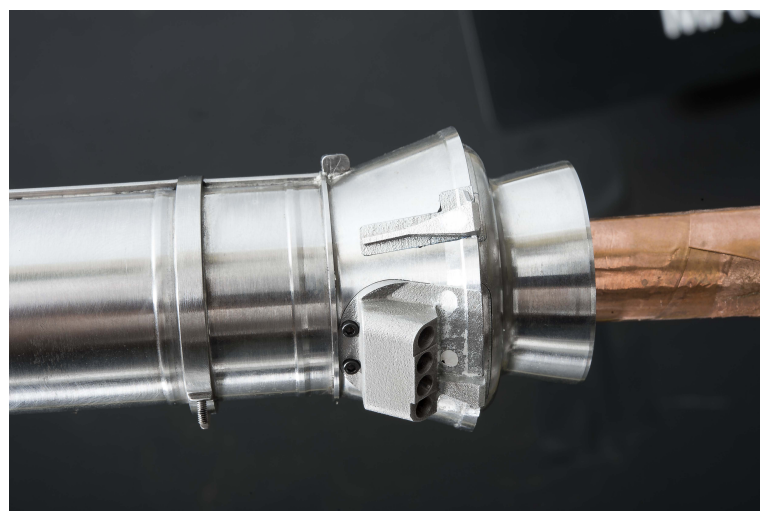

(b) Aft BSM

Fig. 8 Right-hand SRB model BSMs.

\section{Wind Tunnel Data Collection}

The majority of the force and moment data were acquired using the continuous mode of data collection. The core pitch, the SRB pitch, yaw, Y and Z distances from the core, and the SRB high pressure air were set, and data were recorded continuously while the SRBs were translated back in the X (tunnel downstream) direction. Data were scanned at a rate of $30 \mathrm{~Hz}$ in the continuous data acquisition mode for the full time it took the boosters to complete their downstream travel. Continuous data collection was used during the laser light sheet flow visualization portion testing as well. For the PSP and CCDGV testing, wind tunnel data were acquired in move-pause mode. Move-pause data were scanned at a rate of $30 \mathrm{~Hz}$ over two seconds, and the 60 scans of recorded data were averaged before data reduction. The model was not translated during data acquisition.

\section{E. Tunnel Optical Access}

The two tunnel doors, which make up the sidewalls of the test section, can be changed out depending on what type of testing is being conducted. To provide as much optical access as possible during this test, the slotted window doors were installed on both sides of the test section. There is no optical access through the floor or ceiling. The test section doors have nine (9) $5.5^{\prime \prime}$ wide by 48 " tall windows, separated by $1.25 "$ wide webs. The windows are $1.5^{\prime \prime}$ thick, optical quality glass providing minimal distortion for flow visualization techniques. A disadvantage to the webbing is that it limits the field of view of the model, however, it does allow for cameras, lights, and other optical equipment to be attached to the outside of the tunnel. Figure 9 shows the model with CCDGV fiber bundles visible through the east window of the tunnel. The fiber bundles, circled in red, are attached to the webbing with clamps. Figure 10 shows an up close view of the mounting method used for two PSP cameras on the tunnel webbing. 


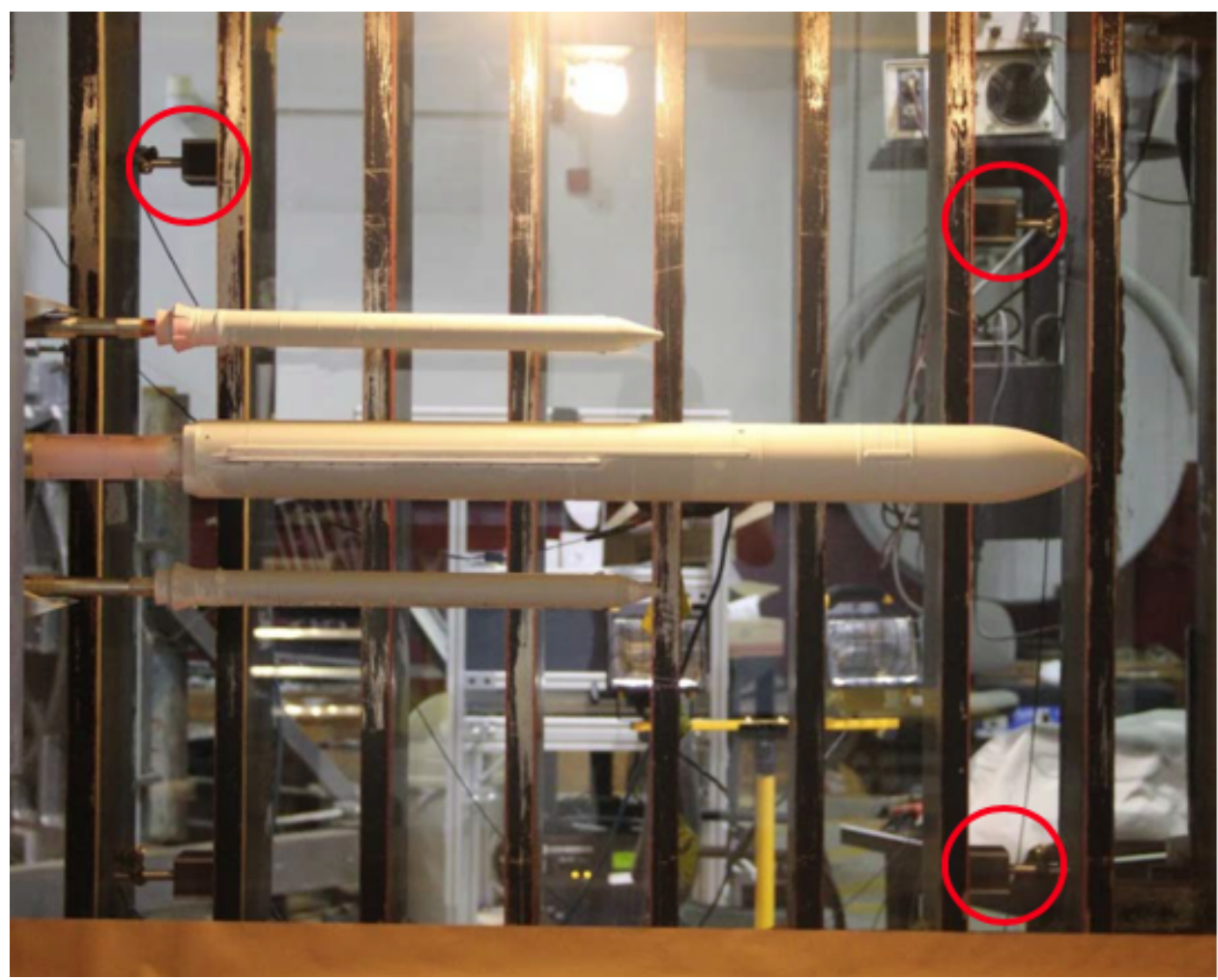

Fig. 9 SLS-27005 with lights and fiber bundles mounted to the webbing on the outside of the test section in the foreground.

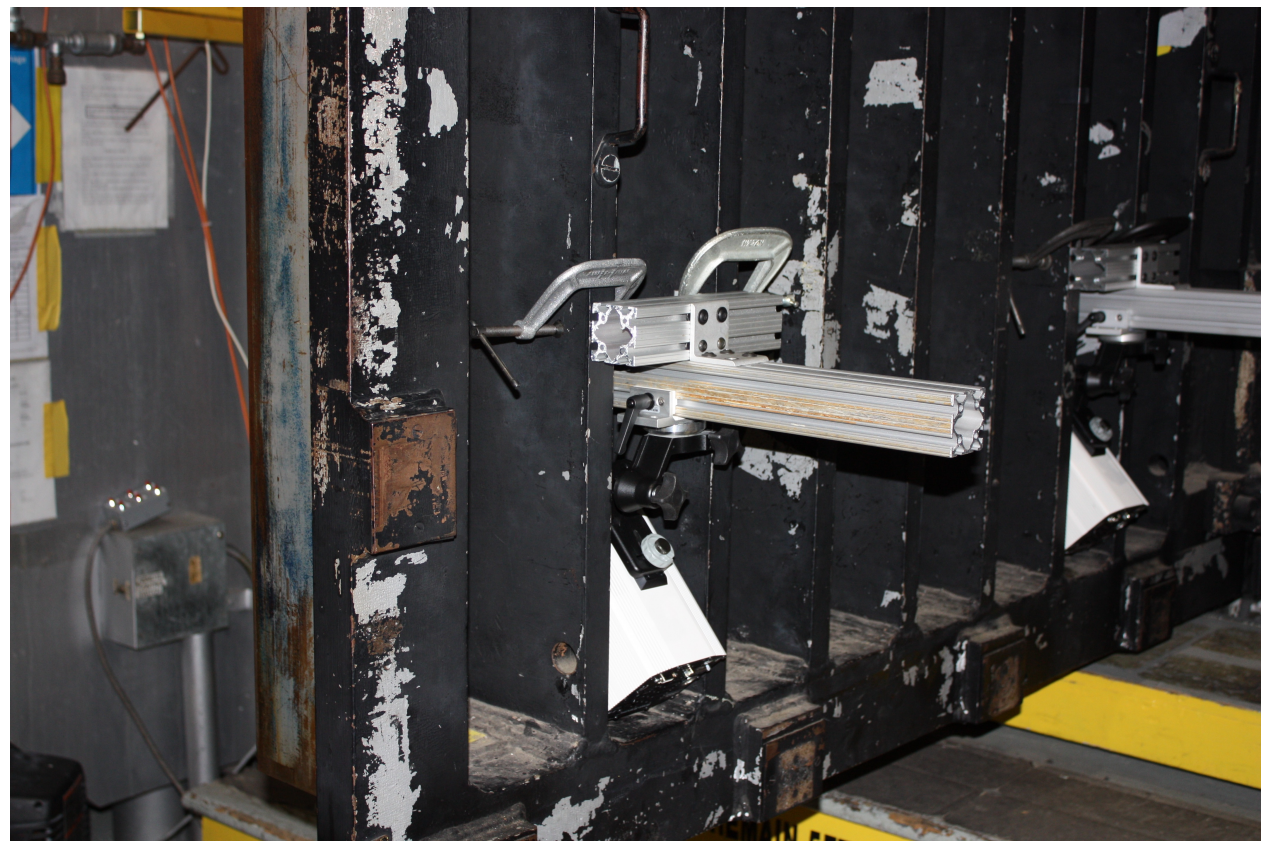

Fig. 10 Mounting of PSP cameras on the tunnel door webbing. 


\section{Numerical Techniques}

CFD solutions for the SLS Block 1B vehicle used in this study were completed after the test itself. This section details the necessary considerations undertaken to provide representative simulations to compare to the wind tunnel database.

\section{A. CFD Run Procedure}

The computational tool used was FUN3D 13.1 [10], a 3D unstructured Navier Stokes solver developed at the NASA Langley Research Center. Each case considered here was simulated at $M=4.0$ to match the wind tunnel freestream conditions. Bulk forces and moments on the vehicle were used to monitor the solution convergence, and the solutions converged rapidly to steady state solution requiring only three to five thousand solver iterations. Turbulence closure was provided using the one equation Spalart-Allmaras[11] turbulence model and the flow was considered fully turbulent. Furthermore, the CFD model was simulated at flight scale, not at the $0.9 \%$ scale used in the tunnel. As such, relevant freestream quantities, such as Reynolds number, and displacements were scaled appropriately in order to match the conditions measured in the tunnel.

A unique volume mesh for each run was created using AFLR3[12]. The surface meshes were created using the ANSA [13] preprocessor and stored independently for the core, LSRB, and RSRB. These three surface representations were then rotated and translated into position automatically using the unique parameters measured in the wind tunnel for each run. Figure 11 shows the three main bodies after the appropriate translations and rotations had been applied for a case with wind tunnel model scale displacements of $d x=0.9$ in., $d y=0.38$ in., $d z=1.47$ in. To match what was observed in the wind tunnel, the displacements in this figure were adjusted to match the scale of the CFD model, which was at flight scale. Finally, the intersect tool from CART3D[14] combined the resulting meshes with farfield boundaries and refinement boxes, which acted as inputs to create the final volume mesh in AFLR3.

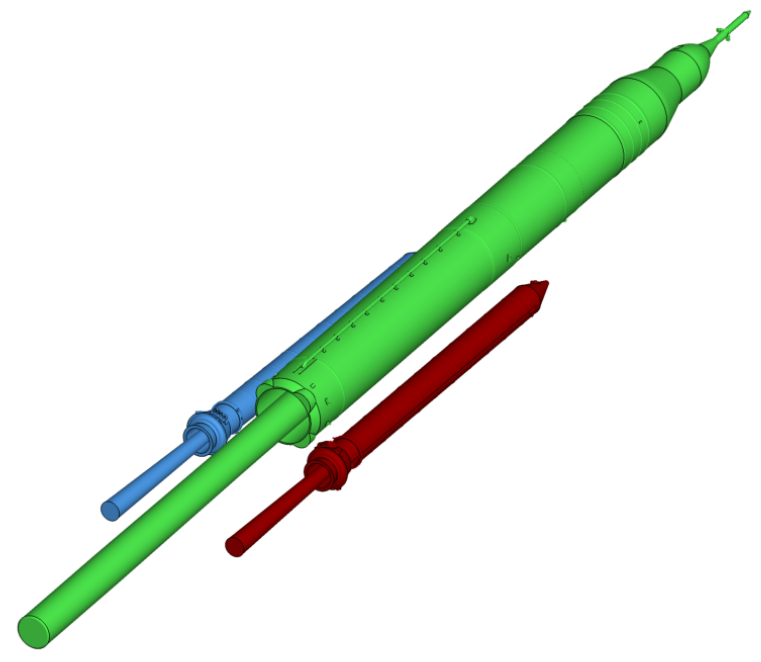

Fig. 11 Relative locations of Core and Boosters with $d x=0.9$ in., $d y=0.38$ in., $d z=1.47$ in.

No slip, adiabatic walls were generally used to model the surface boundaries of the vehicle. For BSM-off cases, the BSM inlet planes were treated as walls; however, to model the BSM plumes (using air), total pressure and total temperature ratios were applied to match the measured thrust coefficient from the experiment. The ratios were calculated using the BSM exit area, BSM exit Mach number, BSM total temperature, and freestream reference conditions.

Feature-based mesh adaption, which seeks to align the mesh with important flow features (i.e., shocks), was utilized for each case. Two rounds of adaptions were generally used for BSM-off cases, while three rounds were necessary to resolve all the transients for the BSM-on cases. Figure 12(a) and Figure 12(b) show a slice of a representative volume mesh near the forebody of the core body before and after three feature-based adaptions had been applied. 


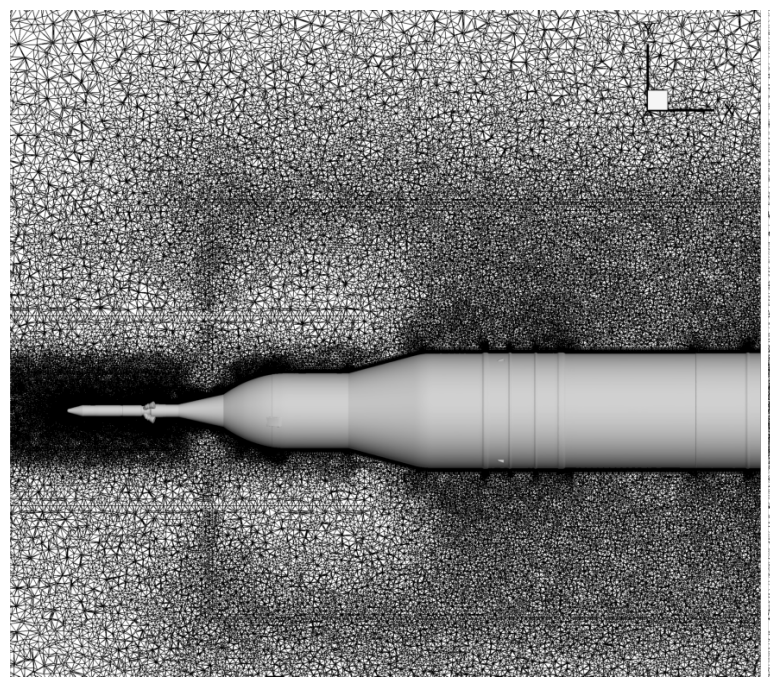

(a) Initial mesh

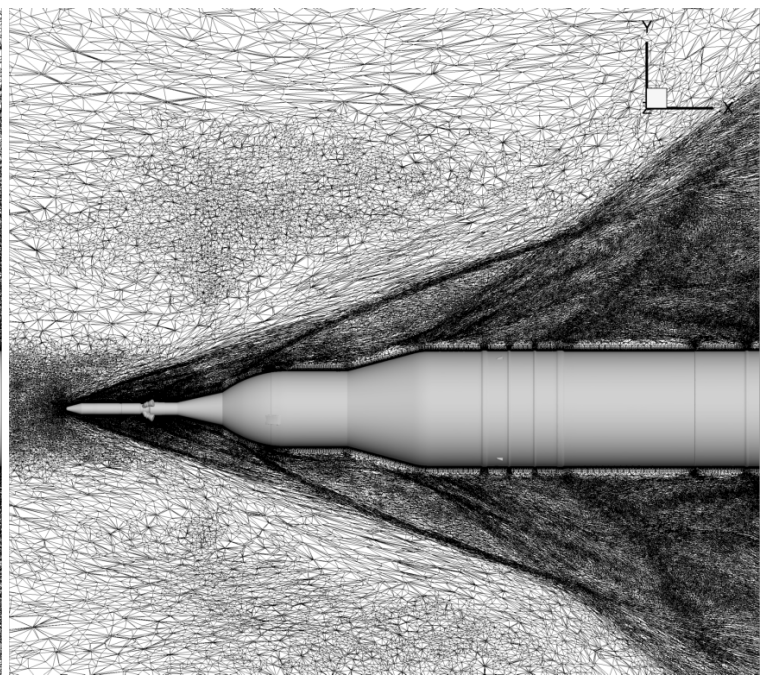

(b) Mesh after three feature based adaptions

Fig. $12 z=0$ volume mesh slice through CORE forebody at $M=4.0$.

\section{B. CFD Run Matrix}

As it would be too costly to simulate the entire wind tunnel test matrix, a method to filter the full matrix down to a representative subsection was necessary. First, the entire matrix was limited to only include discrete values of SRB axial displacement. This step was necessary to reduce a continuous wind tunnel run into discrete, steady-state inputs for CFD. For this work, simulations were undertaken on $d x$ intervals of 0 to 2 inches for BSM-on cases and 0 to 8 inches for BSM-off cases in 0.2 inch increments.

A uniqueness filter was also applied to eliminate runs that were either repeats or "similar" to others in the test matrix. This was done by using the $L_{2}$, or Euclidean, norm of a relevant subset of test variables, namely the translational/angular displacements and thrust settings. Figure $[13$ shows an example of the uniqueness filtering for BSM-on cases at $d x=0.8$ in. This figure shows the unfiltered wind tunnel runs at the fixed $d x$ on the left and the resulting filtered points on the right. Only translational displacement parameters $(d y$ and $d z)$ are shown on these plots; the filtered points take into account many other factors and the resulting plots would look different with other variables on the axes. For the SLS Block 1B crewed booster separation configuration, the resulting CFD matrix consisted of 176 BSM-on and 246 BSM-off cases, which are summarized in Table 2 In this table, all translational and rotational displacements reference the LSRB, hence the use of the subscript $L$. All simulations were performed on the Pleiades and Electra supercomputers at the NASA Advanced Supercomputing (NAS) facility and utilized 1.15 million core hours to complete.
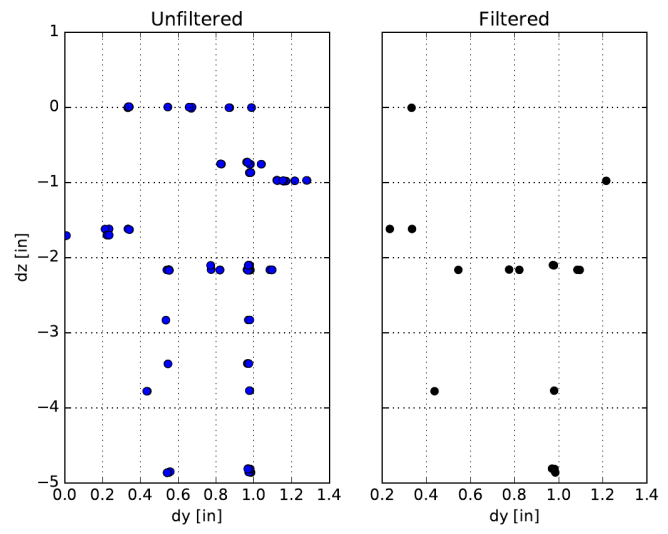

Fig. 13 Filtered run matrix points for BSM-on cases at $d x=0.8$ in. 
Table 2 Tabulated CFD run matrix.

\begin{tabular}{cccccc}
\hline \hline & $d y_{L}$ (in.) & $d z_{L}$ (in.) & $d \psi_{L}\left(^{\circ}\right)$ & $d \theta_{L}\left(^{\circ}\right)$ & $C_{T}$ \\
\hline \hline-1.3 & -2.0 & 0.45 & -1.06 & 0.9 \\
& -1.3 & -2.0 & 0.47 & -1.44 & 1.2 \\
& -0.5 & -1.5 & 0.47 & -1.46 & 1.2 \\
& -1.4 & -0.8 & -1.04 & -1.06 & 0.9 \\
& -0.4 & -0.1 & -0.02 & -1.04 & 0.9 \\
BSM-on & -1.0 & -2.0 & -1.71 & -3.12 & 0.9 \\
& -0.7 & -2.0 & -0.68 & -3.52 & 1.2 \\
$0.0,0.2, \ldots, 2.0$ in. & -1.0 & -2.0 & -1.68 & -3.51 & 1.2 \\
& -0.4 & -1.5 & 0.79 & -3.47 & 1.2 \\
& -0.7 & -3.6 & -0.04 & -7.68 & 1.2 \\
& -1.2 & -3.6 & -1.54 & -7.65 & 1.2 \\
& -1.2 & -4.7 & 0.86 & -5.61 & 1.2 \\
& -1.2 & -4.7 & 0.95 & -7.60 & 1.2 \\
& -1.2 & -4.7 & -0.52 & -7.61 & 1.2 \\
BSM-off & -1.1 & -2.0 & 0.81 & -3.46 & 1.2 \\
& -1.1 & -2.0 & -0.70 & -3.50 & 1.2 \\
\hline $0.0,0.2, \ldots, 8.0$ in. & -1.6 & -2.0 & -1.10 & 0.02 & 0.0 \\
& -0.8 & -6.3 & -0.17 & -6.01 & 0.0 \\
& -0.9 & -2.0 & -0.78 & -1.99 & 0.0 \\
\hline \hline
\end{tabular}




\section{Schlieren}

Schlieren allows the visualization of density gradients in the flow around a wind tunnel model based on the reflection of light, which is directly related to the flow density gradient. This method uses the light refracted along the entire optical path, providing a two-dimensional image of the density gradients in a three-dimensional flow field. Schlieren was used extensively during this test, and during data analysis, since the complex model positioning had the potential of having shocks setup differently depending on how the SRBs were moved into their starting position for a run.

\section{A. Schlieren Setup}

Both test sections at UPWT are equipped with a schlieren system[15]. The system consists of two large spherical mirrors, a light source, knife-edge, optical beam splitter, Canon 5DS still camera, high definition video camera, flat mirror, and an image screen. Figure 14 shows a sketch of the UPWT schlieren system setup. The entire system is supported by a beam on the ceiling, and can be moved as a single unit along the longitudinal axis of the test section. A xenon vapor arc lamp is used to provide a continuous light source. An optical beam splitter is located just behind the knife edge to send the schlieren image to both the video and still camera. Run information is overlayed on both the still and video images. The schlieren system is operated continuously during testing when optical access is available. During this testing effort, schlieren was used throughout all of the testing except during CCDGV and PSP because the two setups prevented adequate optical access for the schlieren system.

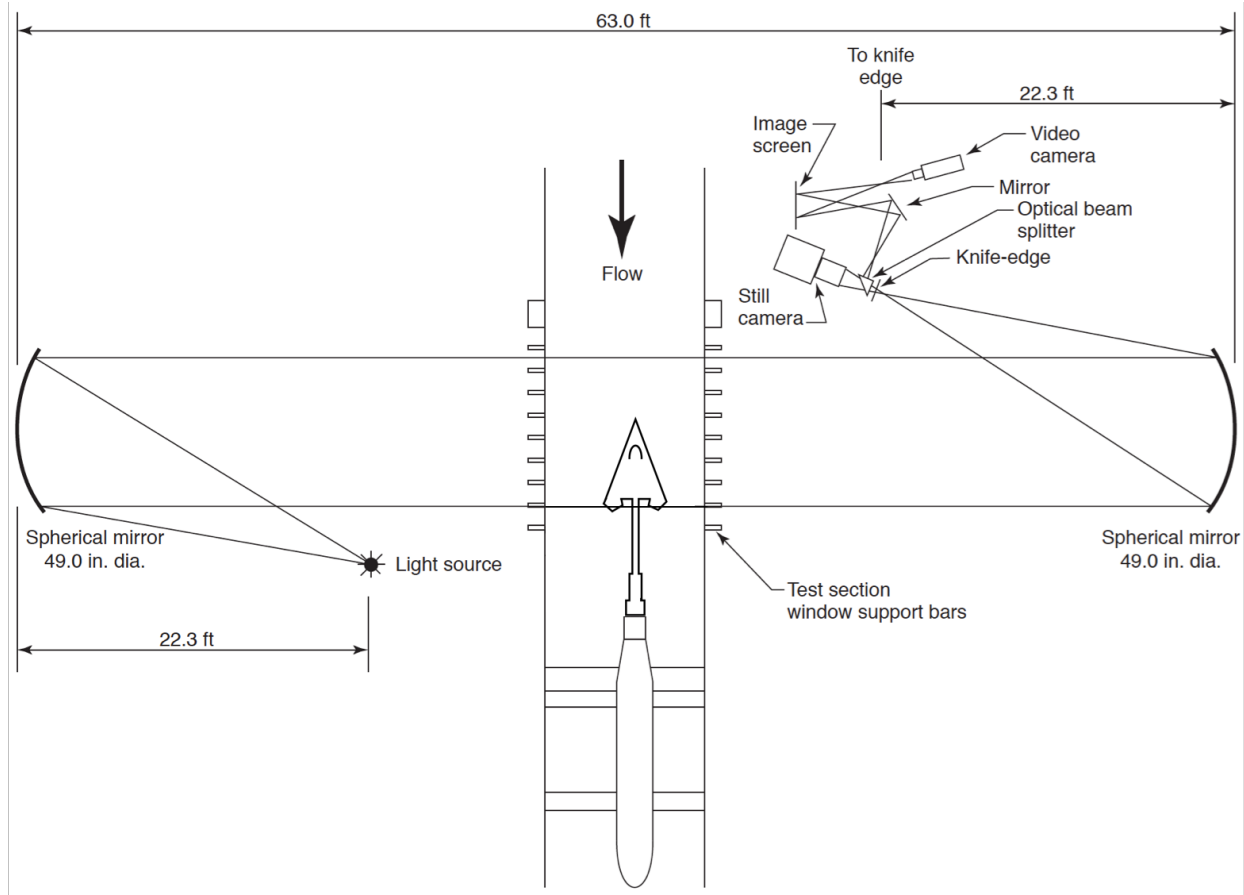

Fig. 14 Sketch of the LaRC UPWT schlieren system.

\section{B. Schlieren vs. CFD Results}

Figure 15 shows a schlieren image on the left taken during wind tunnel testing and the corresponding density gradient slice from the CFD solution at the $\mathrm{Z}=0$ plane for the same model position and tunnel conditions. Note that in the schlieren image, the vertical lines are the webbing on the tunnel doors. The effects of the high pressure air blowing out of the forward and aft BSM nozzles are evident in both images. The tip of the core nose is cut off from view by the front of the tunnel door, but all other shocks line up between the two images. The coalescing or merging of the shocks off of the Orion Multi Purpose Crew Vehicle (MCPV) and the Universal Stage Adapter (USA) is hidden behind one of the webbing bars in the schlieren image, but the interaction of the two shocks can be seen in relation to one another in Figure 16, showing progressive schlieren images as the SRBs move back in relationship to the core stage. 


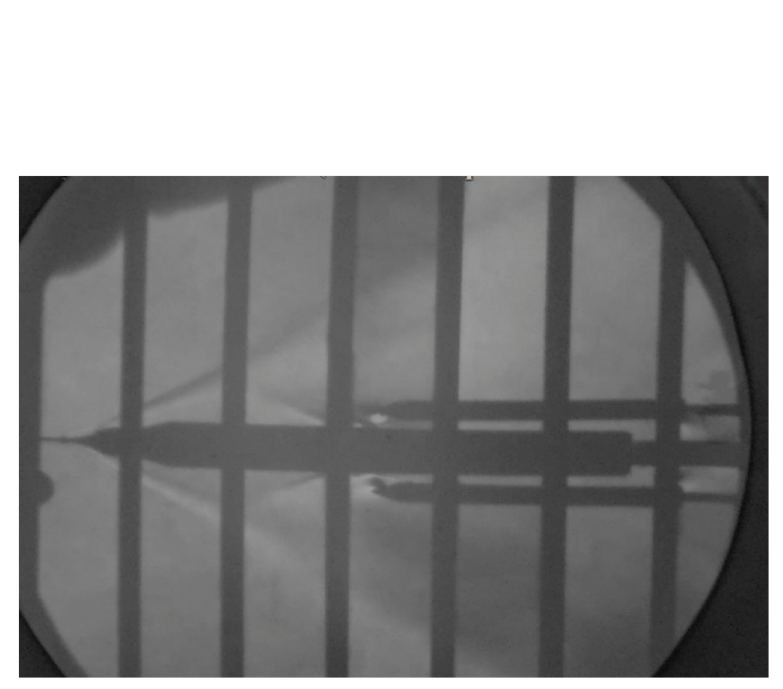

(a) Schlieren image

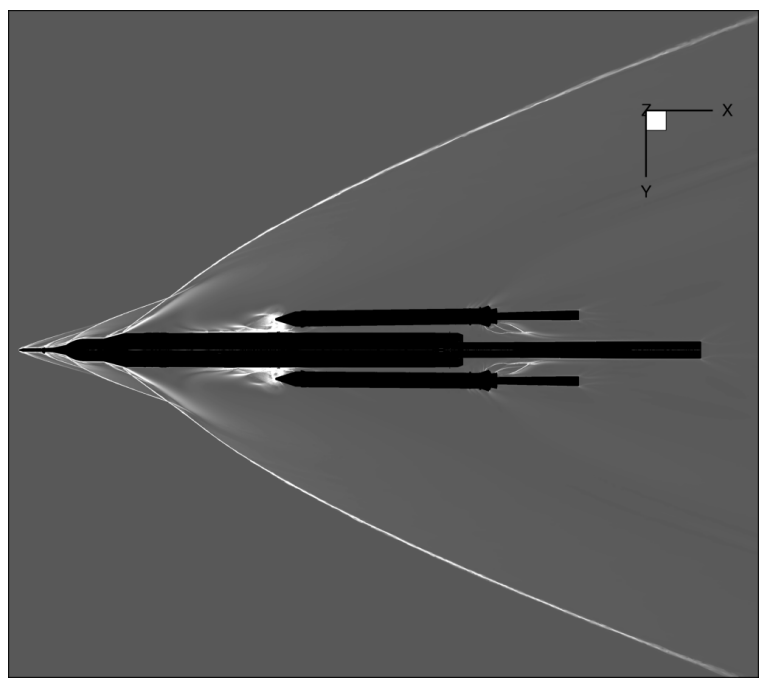

(b) CFD density gradient $\mathrm{Z}=0$ cut

Fig. 15 Schlieren image from tunnel testing versus a CFD density gradient solution at the same model location, Mach = 4.0.

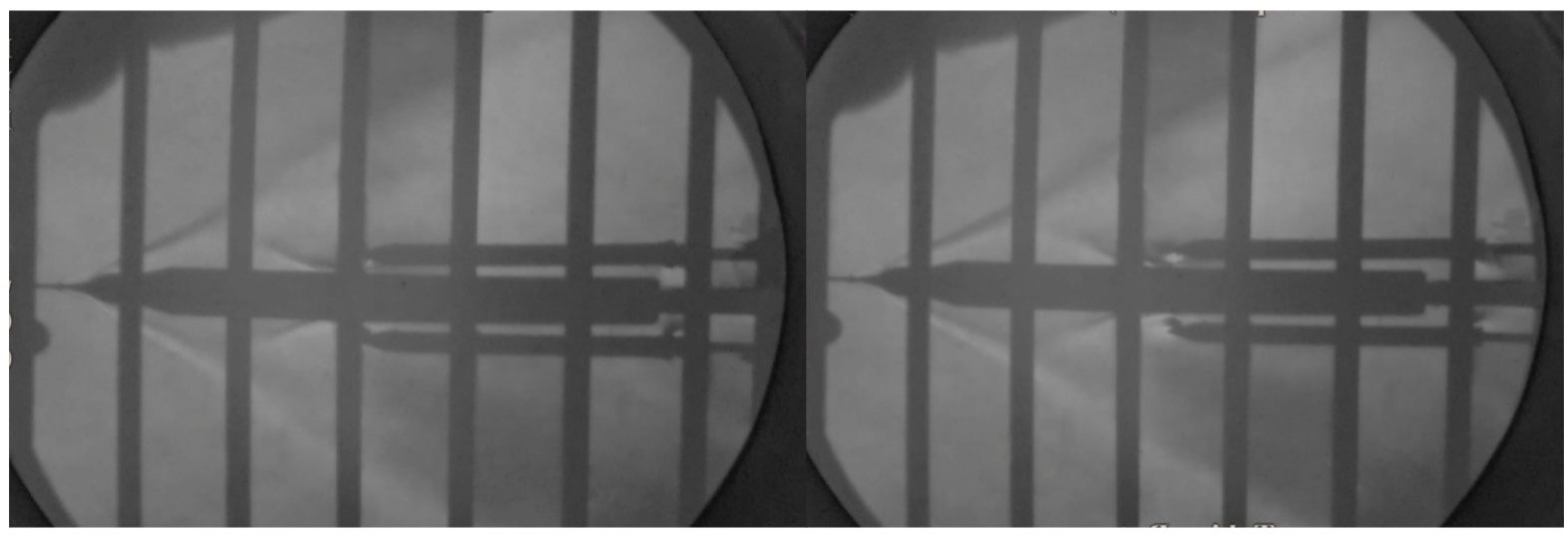

Fig. 16 Wind tunnel test schlieren images, as the SRBs move further back in relation to the core.

\section{Cross-correlation Doppler Global Velocimetry}

Cross-correlation Doppler Global Velocimetry was done during a portion of testing to obtain three-velocity component flow measurements in regions of interest. A secondary goal was to demonstrate CCDGV capabilities in the LaRC UPWT. For more detailed information on the CCDGV method used during this test, see the work by Lowe[16].

\section{A. CCDGV Setup}

Most of the CCDGV testing was completed using an approximately 2-4mm diameter laser beam. The use of a laser sheet was attempted, but the area between the left hand booster and the core body was small, causing the sheet to reflect off of the model and corrupt the data. For certain model positions, the laser was traversed to 3 or 4 different heights (model Y direction) between the left hand booster and the core body to get a more complete data set of the flow. The laser was set at a Y height between the left hand booster and the core, an X distance back on the core/SRB, and traversed along a path 3 to 7 inches long in the $\mathrm{Z}$ direction. Figure 17 shows a laser beam positioned for testing and the model coordinate system. Three fiber bundles, each with 50,000 fibers, were used for imaging. Two of the bundles were upstream of the laser, and one bundle was downstream of the laser. Data were recorded from each of the three bundles simultaneously allowing for the calculation of all three velocity components. A fourth fiber bundle was initially setup 
downstream of the laser, but was damaged during setup, and was unusable throughout the experiment. See Figure 9 for fiber bundle locations, circled in red.

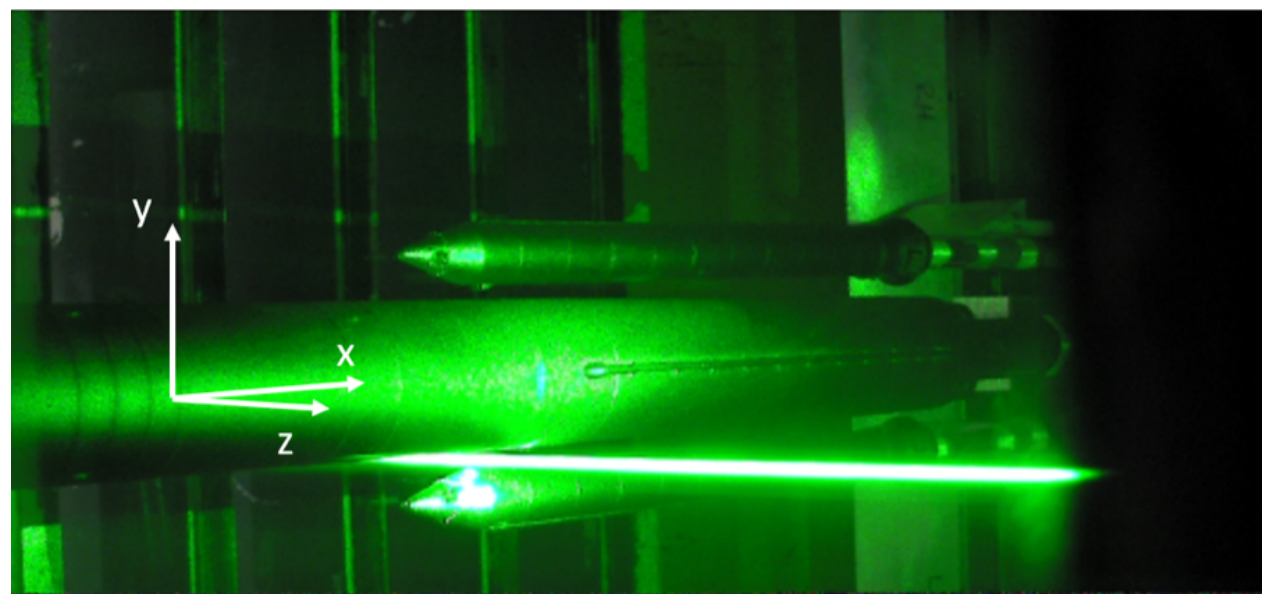

Fig. 17 Laser beam between the left hand SRB and the core stage, with the model coordinate system overlayed.

A Coherent Verdi 18 Watt continuous wave Nd:YVO4 laser was directed through a window on the port-side door (pilots view in a standard model setup, also referred to as the east door). The fiber-laser-produced images were each relayed to two 5.5 MPix sCMOS scientific complementary metal-oxide semiconductor cameras (PCO.edge brand), and LabVIEW was used to control automatic scanning of the laser frequency during data acquisition. The light from the fiber bundles was split into two paths and imaged by the cameras: one of the cameras viewed the scattering through an iodine gas vapor filter while the second camera did not. The first camera is sensitive to the Doppler shift of the light while the second camera acts as an intensity reference. Typically, 72 images were recorded per data set, each image at a different laser frequency. Camera exposure time varied between 500 and 2000 milliseconds depending on seeding and laser flare. The tunnel was seeded with water through a manually controlled system. For optimal seeding, the dew point inside of the tunnel was kept between $25^{\circ} \mathrm{F}$ and $40^{\circ} \mathrm{F}$ for this experiment.

The Mie scattered light was sent through the iodine cell and its frequency transduced to intensity. The scattered lights transmission spectrum signal is cross-correlated to a reference transmission signal, which is used to estimate the Doppler shift from which the flow velocity can be calculated.

For this test, we were unable to seed the flow through the SRBs. The high pressure air used for the SRBs was very dry, and therefore, did not have many water/ice particles to scatter the light. Data were not able to be collected in the flow where the high pressure air from the SRBs was, so there are missing segments within the data. This could possibly lead to bias error in the velocity data where the velocities are weighted toward the velocity in the seeded fluid, particularly in unsteady separated regions of the flow.

\section{B. CCDGV Data vs. CFD Results}

Three CFD solutions were run that could be compared to the CCDGV results. The CFD solutions where run at full scale, without walls, so the results are not direct comparisons. It was found that for the runs in which high pressure air was flowing through the SRB nozzles, the results did not compare well. As discussed previously, CCDGV data acquisition was difficult in the BSM plumes due to the lack of seeding, so a good comparison to CFD solutions would not be expected. Figure 18 shows the constant X slice of the CFD $u / U_{\infty}$ velocity vector solution, the normalized $u$ velocity component, with the two constant $Y$ cuts where data was extracted to match the location of the laser in the tunnel. The solution is for a case in which the boosters were offset from the mated position on the core in X, Y, and $\mathrm{Z}$, and the high pressure air was off. The freestream condition was at a Mach of 4.0 and Re of $1.25 \mathrm{million} /$ foot (see Table 11 .

Figure 19 is a plot of the $u$ velocity component (X direction, into the page) of the flowfield divided by the freestream velocity $\left(U_{\infty}\right)$ of the CCDGV data taken in the tunnel and the CFD solution taken from the cuts in Figure 18 . Both cuts are between the main core stage and the left hand booster. Cut 1 is closest to the core, and cut 2 is closer to the left hand booster. The black circles represent the core and the two boosters for reference (left hand booster is below the core, right hand booster is above), and the laser/cut 2 is located at zero Y relative to the core and boosters. The CCDGV data are 


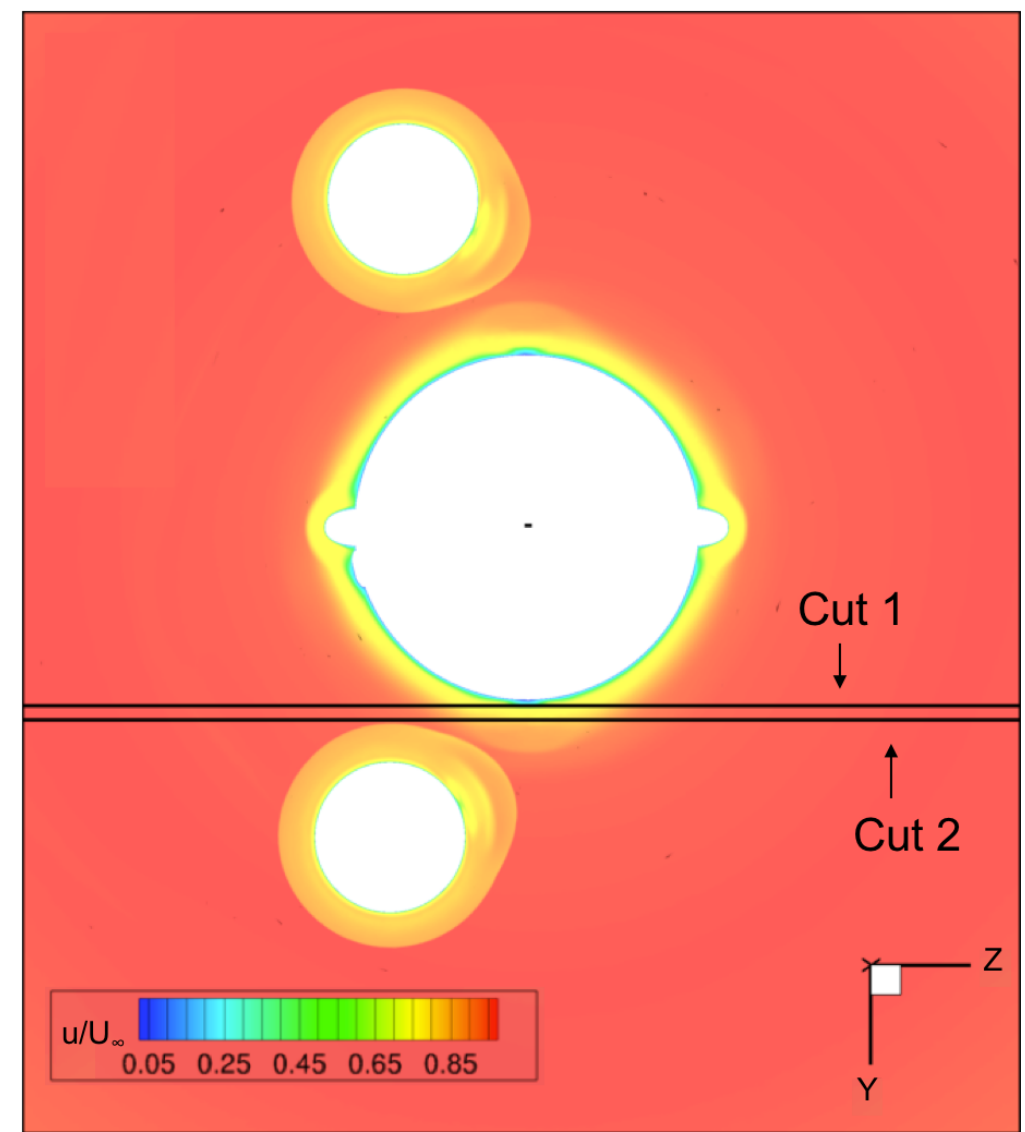

Fig. 18 CFD X slice, with two Y cuts taken of the $u / U_{\infty}$ velocity vector.

filled in symbols with repeat points plotted in the same color but different symbols, and the CFD slices are solid lines. It can be noted that even the repeat CCDGV data does not fall on top of each other because of the instability and dynamics in the area. While both CFD Y cuts follow the trend of the CCDGV data, both are slightly shifted up (i.e., have higher predicted velocities). This could be due to the fact that the CFD was run with no walls. CFD cut 2 has a much sharper and larger peak than any of the other data. As can be seen in Figure 18, just a small move in the positive $\mathrm{Y}$ direction can make a large difference in whether the data are being taking in the boundary layer or not.

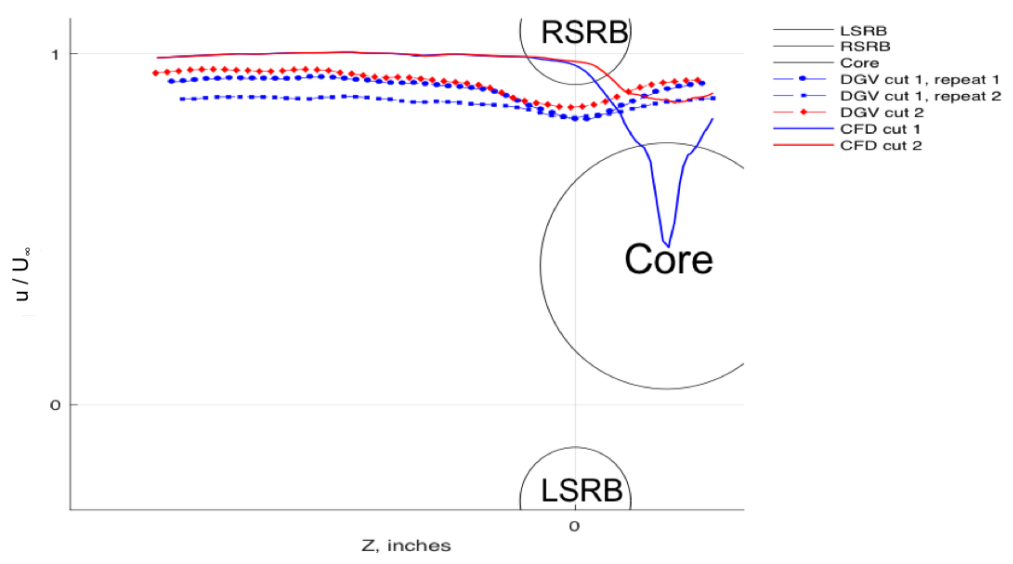

Fig. $19 u / U_{\infty}$, CCDGV vs. CFD. 
Figure 20 shows the same data as Figure 19, except it is the $v$ velocity component (Y direction, positive up) of the flowfield divided by the freestream velocity. The CCDGV cut 1 data showed a slight increase in the $v$ velocity in the area between the core and the LSRB that the CFD did not see. The cut 2 CFD data shows a slight negative velocity in a small region just below the core. Again, small variations can be due to the difference in the CFD solution being run at full scale with no walls, and the CCDGV being run on a scaled model in a wind tunnel.

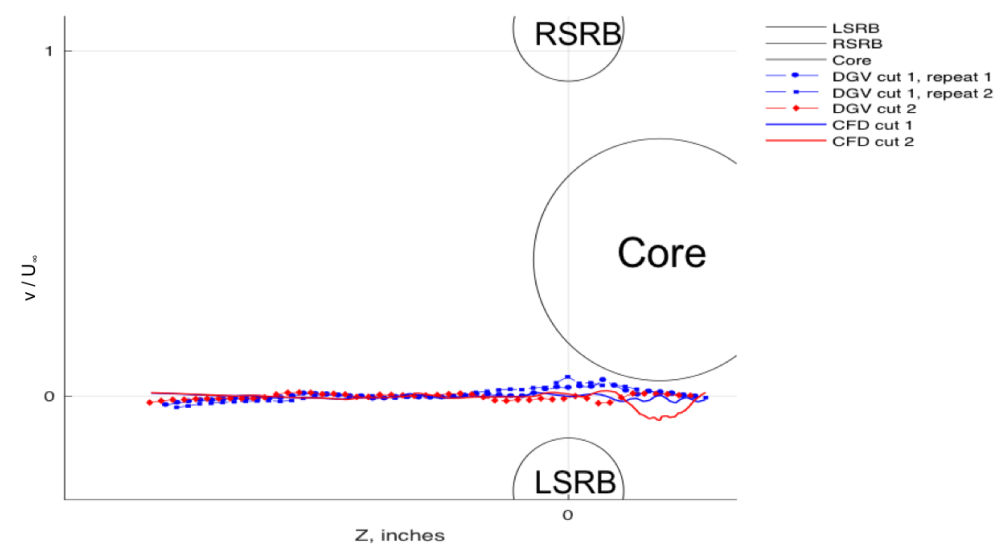

Fig. $20 v / U_{\infty}$, CCDGV vs. CFD.

Figure 21 shows the same data as Figures 19 and 20 , except it is the $w$ velocity component ( $\mathrm{Z}$ direction, positive right) of the flowfield divided by the freestream velocity. All of the data show the $w$ component of the velocity to be close to zero away from any influence of the SLS core stage or the LSRB. The CCDGV data show a slight rise in the $w$ component of the velocity above the LSRB followed by a sloped return to zero. The CFD shows a small jump to a positive velocity, followed quickly by a jump to a negative velocity near the back side of the LSRB.

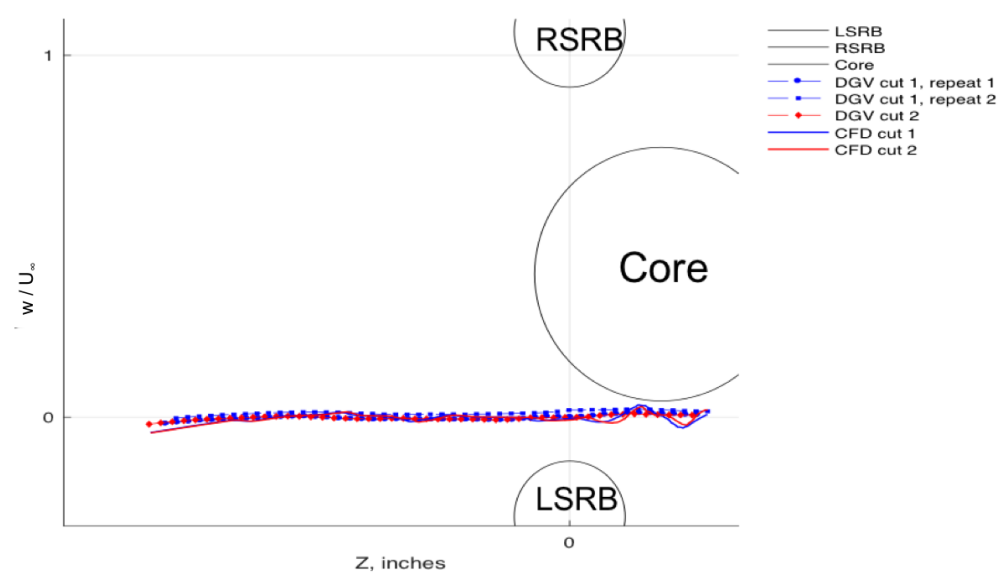

Fig. $21 w / U_{\infty}$, CCDGV vs. CFD.

\section{Laser Light Sheet Flow Visualization}

Flow visualization was done between the core and the left hand SRB. A continuous wave green laser sheet was directed at the model through the port-side window, and illuminated ice crystals in the flow, showing some flow phenomena including shock waves. For more information on the background, testing, and setup see Danehy [17]. 


\section{A. Flow Visualization Setup}

After CCDGV testing was completed, the CCDGV equipment setup was reconfigured to perform laser light sheet flow visualization. The laser beam was directed through a single negative cylindrical lens, which expanded the beam into a vertical planar sheet perpendicular to the streamwise axis of the flow, and then through the port-side window of the tunnel. The tunnel flow was seeded with water in a controlled manner, water entered the tunnel through a tube on the ceiling, behind the test section, about 10 feet downstream of the second minimum in the tunnel. The tunnel is continuously replenished with dry air, so the water seeding needed to be repeated multiple times. The water seeding level and the laser intensity were adjusted before runs to maintain a high enough level of scattered light intensity while not saturating the camera.

The laser sheet reflecting off of the ice crystals resulted in Mie scattered light, which was collected with two separate camera systems. Figure 22 shows one camera view of the laser light sheet flow visualization at a Mach of 4.0, between the left hand SRB and the core stage of the model, with no high pressure air being run through the BSMs. The two SRBs, the core stage, laser light reflecting off of the model, three separate shock waves, and a shadow are all labeled in the figure. The graphic overlay on the tunnel displays the Mach number, unit Re (million/ft), HO (freestream stagnation pressure, psfa), the date, time, test number, run number, point number, tunnel dew point $\left({ }^{\circ} \mathrm{F}\right), \psi_{C R H}$ (right hand SRB yaw angle relative to the core, ${ }^{\circ}$, left hand SRB yaw angle would have the equal but opposite value), $\theta_{S R B}$ (SRB pitch relative to the core, ${ }^{\circ}$, measurement was from the right hand SRB, but both SRBs would be at the same angle), and $\theta_{C R}$ (core pitch angle, ${ }^{\circ}$ ). The overlay also shows the $\Delta X_{R}, \Delta Y_{R}$, and $\Delta Z_{R}$ locations of the right hand SRB relative to the mated position on the core, but those numbers are blacked out for SBU purposes. The RSRB is above the core stage, and the LSRB is below the core stage in the picture. A shadow is seen behind the LSRB, where the light from the laser is blocked by the SRB itself. The laser reflecting off of the ice particles in the stream created more illumination when there were more ice crystals to reflect off, and less illumination of the laser sheet when there were fewer particles. Since shock waves create a density change, the laser light allows for visualization of shocks in the flow.

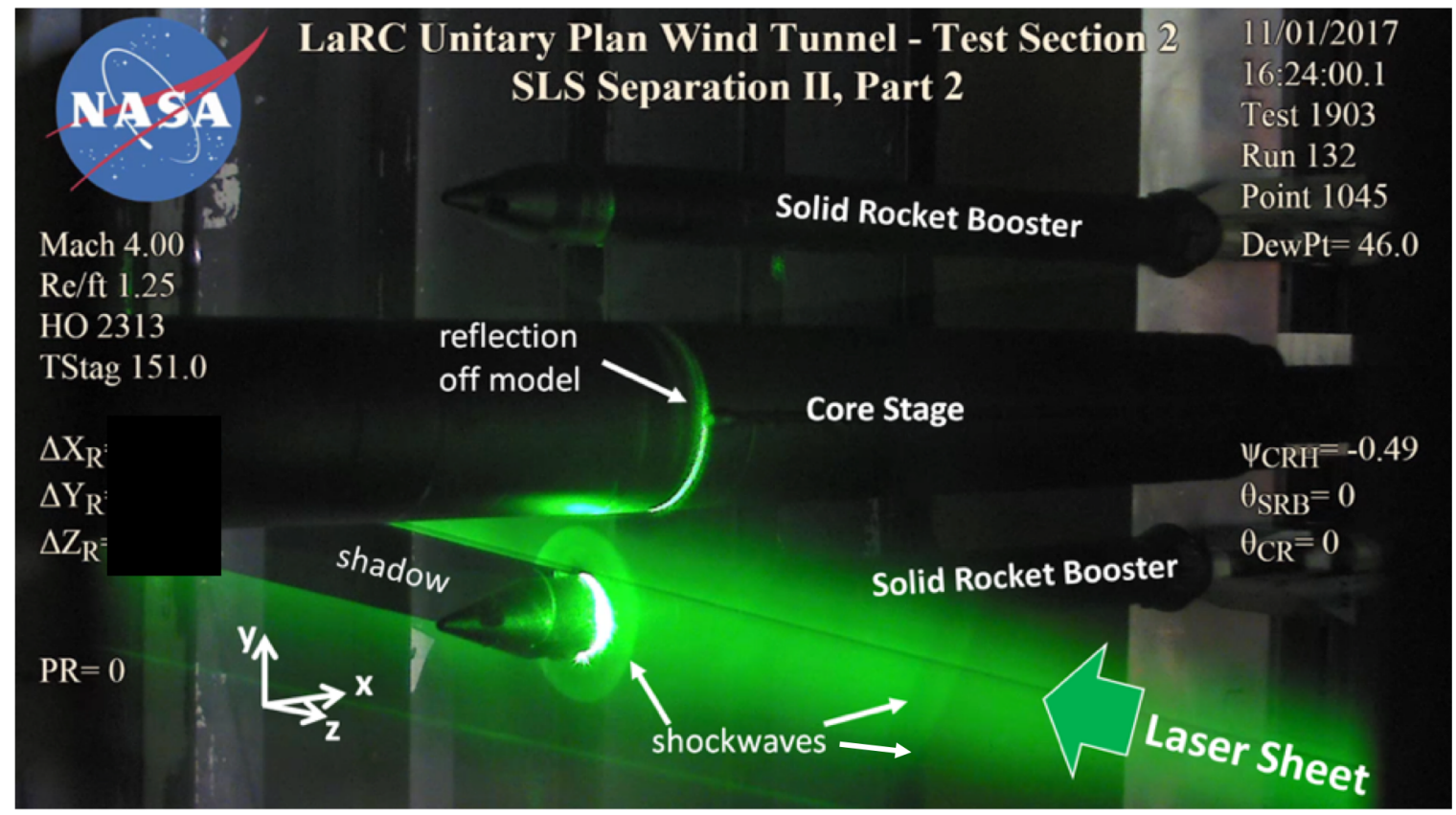

Fig. 22 Laser Light Sheet Flow Visualization.

\section{B. Flow Visualization Results vs. CFD Results}

Figure 23 shows the camera view of the SLS separation model with the laser light sheet being used for flow visualization on the left, and the CFD simulation of the model in the same orientation and at the same flow conditions with an $\mathrm{X}$ slice showing the density at the same location as the laser sheet in the tunnel camera view on the right. Unlike with the CCDGV CFD comparison data, the flow visualization comparison CFD was run at wind tunnel scale. This run was with the SRBs at a certain $\mathrm{X}, \mathrm{Y}$, and $\mathrm{Z}$ separation distance, and a slight $\left(-0.49^{\circ}\right)$ yaw angle, with the high pressure 
air through the BSMs off. Freestream conditions were at a Mach of 4.0 and a Re of 1.25 million/foot. The shock waves, which are labeled in the camera view, can be seen as a lightening of the laser reflection. The shock waves in the CFD density slice are black circles. Qualitatively, the shock locations on the camera match up with the shock locations in the CFD data. There is one shock cone coming off of the nose of the SRB, and two shocks coming from the forward portion of the core stage.

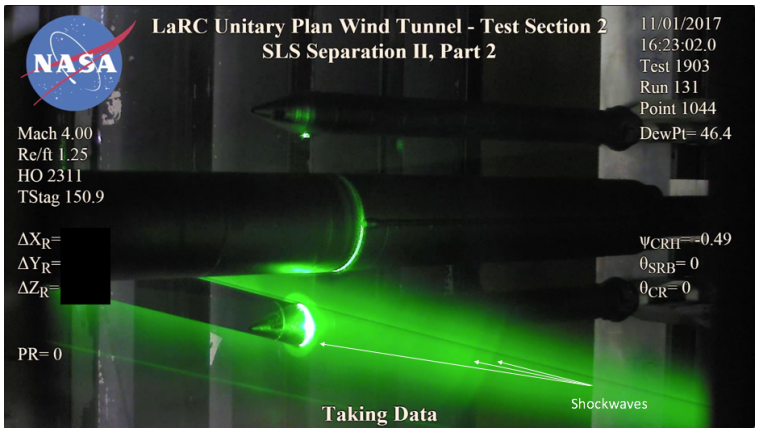

(a) Flow visualization camera view

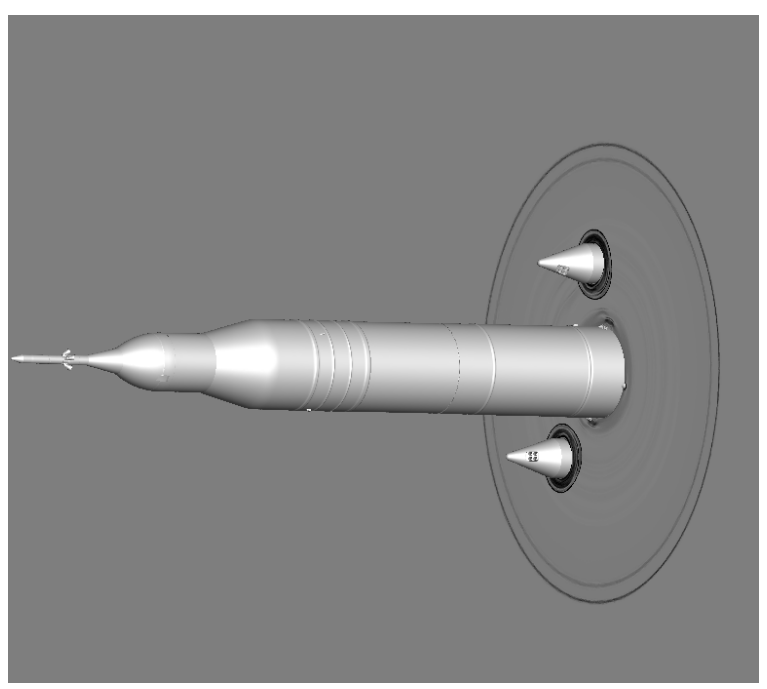

(b) CFD density cut

Fig. 23 Flow visualization camera view vs. CFD with no high pressure air blown through the SRB nozzles.

Figure 24 is the same as Figure 23 above, but with the SRBs further back in relation to the core stage. The figure shows the camera view of the SLS separation model with the laser light sheet being used for flow visualization on the left, and the CFD simulation of the model in the same orientation and at the same flow conditions with an X slice showing the density at the same location as the laser sheet in the tunnel camera view on the right. Again, qualitatively, the shock locations on the camera match up with the shock locations in the CFD data. There are two shock cones coming from the forward nose area of the core stage.

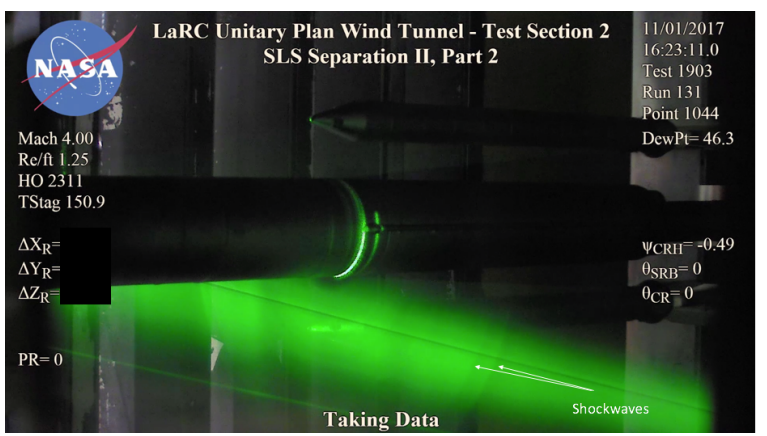

(a) Flow visualization camera view

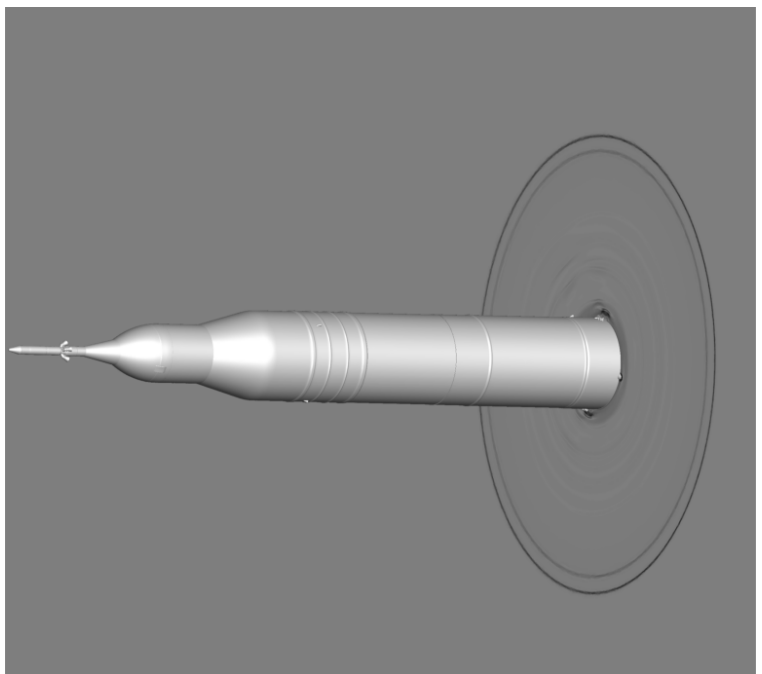

(b) CFD density cut

Fig. 24 Flow visualization camera view vs. CFD with no high pressure air blown through the SRB nozzles. 
Figure 25 is the same model position, laser position, and tunnel conditions as Figure 24 above, but with high pressure air flowing through the BSM nozzles (1500 psi in the SRB sting plenum). The effect of the high pressure air being blown through the forward BSM nozzles can be seen in both the flow visualization camera view (Figure 25(a)], and in the CFD density results (Figure 25(b)). The high pressure air was not seeded for this test, so it was thought that there would be nothing for the laser to reflect off in the areas with a high concentration of BSM nozzle flow, however, some scattering is visible at the exit of the nozzles. The scatter may be from condensed $\mathrm{O}_{2}$ or $\mathrm{N}_{2}$ in the jets, or possibly condensation of the very small amount of water vapor still in this dry air.

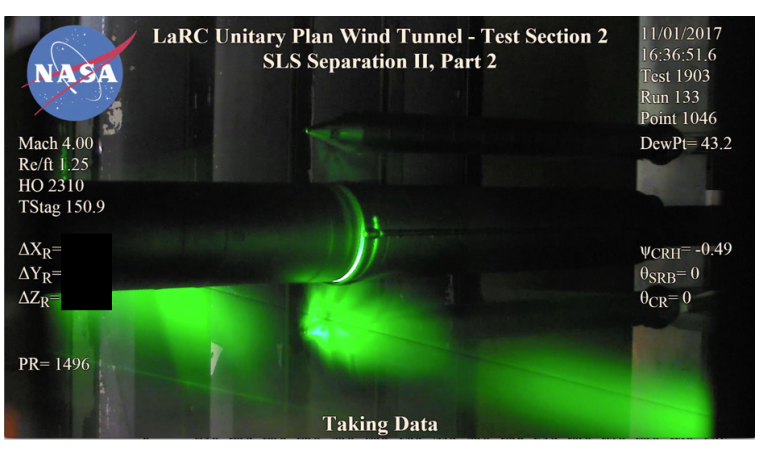

(a) Flow visualization camera view

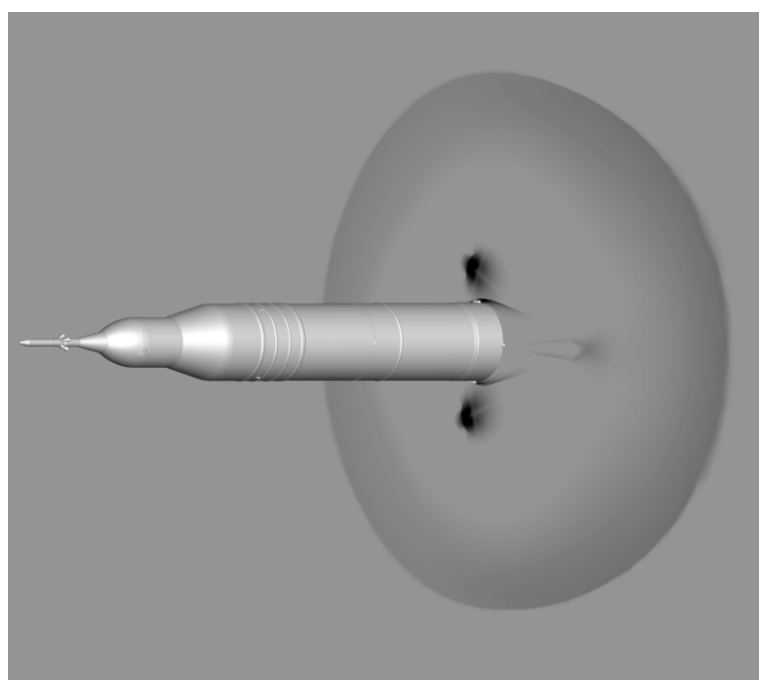

(b) CFD density cut

Fig. 25 Flow visualization camera view vs. CFD with high pressure air blown through the SRB nozzles.

Figure 26 shows zoomed in views of the jets exiting the left hand SRB forward BSM nozzles. The flow visualization camera view, Figure 26(a) was taken with the SRBs in a more aft position than in Figure 25, but the laser in the same position. The dark area is the air with no seed particles, while the bright area is condensing $\mathrm{O}_{2}, \mathrm{~N}_{2}$ or $\mathrm{H}_{2} \mathrm{O}$ just existing the nozzles. Figure 26(b) is the same slice as Figure 25(b) at a different angle to allow a better view of the forward BSM left hand SRB jets exiting the nozzles.

Conventional schlieren, utilized in the Langley UPWT, provides a two-dimensional image of the density gradients in a three-dimensional flow field. The use of the laser sheet provides a two-dimensional image of the same flow in the perpendicular dimension that is missed with schlieren, so the two techniques used in conjunction can give a more complete view of the flow and shock waves around a model.

\section{Pressure Sensitive Paint}

Global static pressure surface measurements can be done on a wind tunnel model using PSP[18]. In a typical application, a PSP coating is illuminated using blue or UV light, which causes the dye in the coating to luminescence. The intensity with which the PSP luminesces varies inversely with the amount of oxygen present locally. Since oxygen is a fixed mole fraction of air, the amount of oxygen present directly relates to the pressure, and therefore, the local pressure can be determined by measuring the intensity of the emitted light from the paint on the model.

\section{A. PSP Setup}

Three layers of paint were applied to the model: a primer/basecoat layer, a screen layer, and the PSP coating. The primer/basecoat layer was applied to not only protect the model from the PSP, but also to enhance the adhesion of the PSP to the metal surface. A screen layer was then applied to the primer to reduce the effect of the primer on the PSP coating. Finally, the oxygen permeable PSP coating was applied. The next step involved curing the PSP layer at $65^{\circ} \mathrm{C}$ for at least one hour, required for optimal performance of the PSP. Eight cameras, four on each side of the test section, were positioned to record all parts of the model. A multitude of LED-based lights configured to run in the 


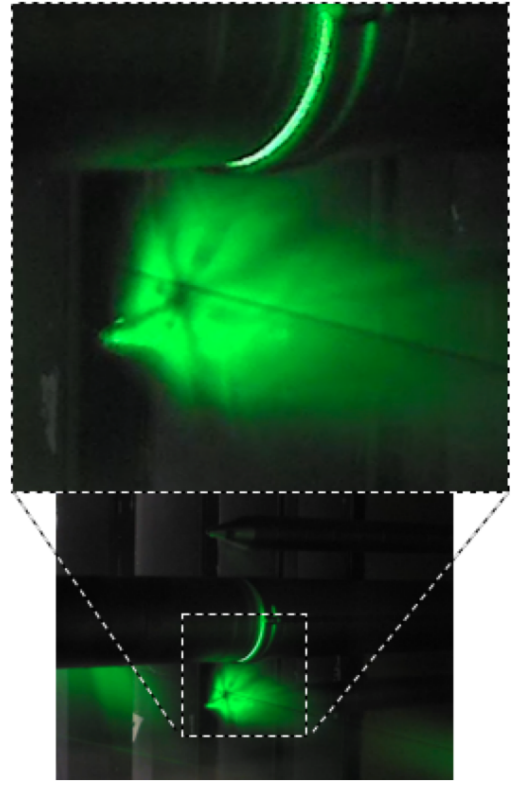

(a) Flow visualization camera view with close up shot of forward LSRB BSM high pressure air jet

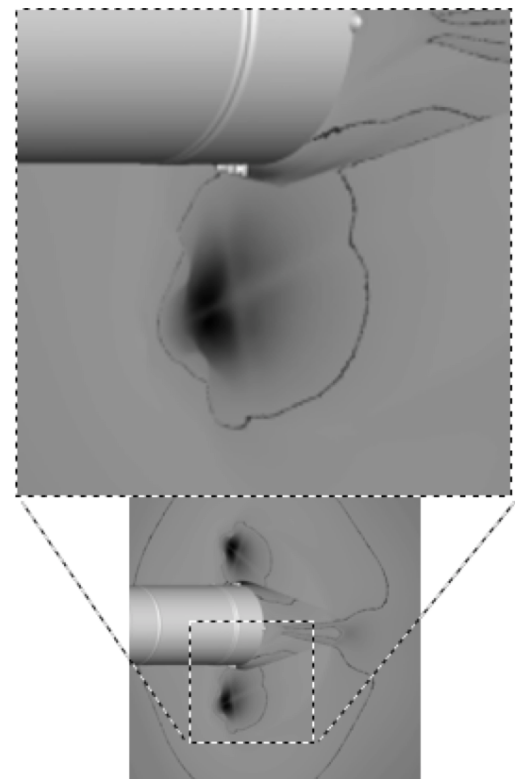

(b) CFD density cut with close up shot of forward LSRB BSM high pressure air jet

Fig. 26 Flow visualization camera view vs. CFD density cut with close up of the high pressure air blown through the SRB BSM nozzles.

"lifetime-mode" [18] were also attached to both sides of the tunnel. Prior to acquiring PSP images at run conditions, a correction was performed to account for paint application inconsistencies and dye inhomogeneity in the PSP coating. This was done by acquiring wind-off images at different pressures in the facility, encompassing the expected pressure range throughout the wind-on testing[19, 20]. A single static pressure tap on both noses was used to anchor the PSP measurements. For mapping the PSP images to a surface grid, black dots were applied to the PSP coating, whose positions were measured precisely using a FARO Arm[21]. These dots were then cast into the coordinate system of the surface grid. The data analysis programs used for PSP could accurately account for the independent model motion of the boosters using the measured position coordinates provided by the wind tunnel operating system[22].

\section{B. PSP vs. CFD Results}

Figure 27 shows comparison images of the PSP data taken in the tunnel and the CFD prediction for a case with no high pressure air being run through the BSMs. Because of the way the run matrix was filtered for CFD, the delta X position of the SRBs did not match up exactly with the test point, and the SRBs are slightly further back in relation to the core in the CFD run than in the PSP test point. The PSP qualitatively follows the CFD prediction, though it does consistently measure a lower pressure than the CFD predicts. Note that the PSP data on the stings is invalid due to poor lighting in that region and less than optimum imaging from the cameras resulting from the high curvature.

Figure 28 shows a plot of $\mathrm{X}$-position on the model vs. $C_{p}$ (note that $\mathrm{X}=0$ is ahead of the Launch Abort System (LAS) nose) for the PSP and CFD data. The PSP and CFD $C_{p}$ values were extracted along a line of 100 points on the core using the same X-, Y-, and Z-coordinates. Spatially, the PSP data (blue line) and CFD data (black line) are in good agreement with the pressure peaks and valleys, lining up well. However, the PSP data is consistently lower than the CFD data. A correction factor was determined by comparing the measured pressure at the static pressure tap (located on the USA) with the predicted CFD pressure at the same location. This difference was then subtracted from the CFD data as a scalar offset to provide the "corrected" CFD data (orange line). The corrected CFD data agrees much better with the PSP data but there are still some differences, most notably at the trailing edge of the core (higher X values).

Figure 29 shows comparison images of the PSP data taken in the tunnel and the CFD prediction for a case with high pressure air being run through the BSMs. To account for the change in pitch angle of the SRBs, the PSP data analysis program had to treat these as a static deflection of the component. This was used to create a deformed grid of the 


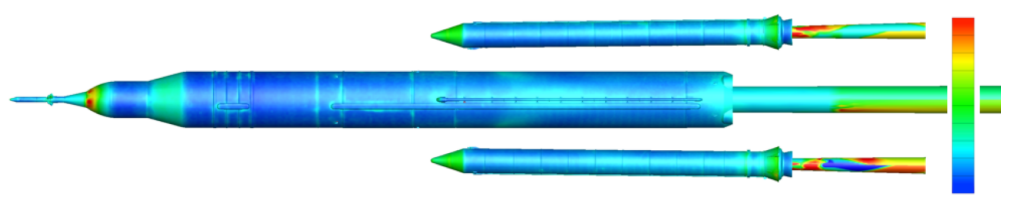

(a) PSP front view with no high pressure air flow through the BSMs, $C_{p}$

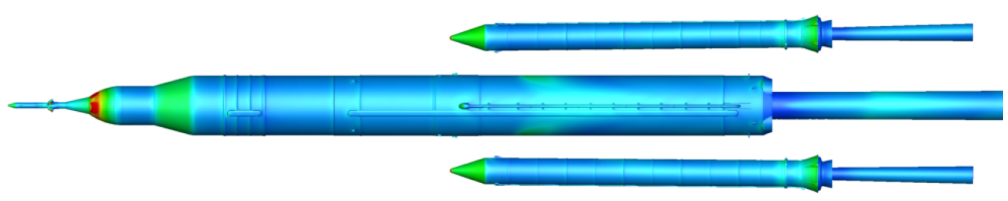

(b) CFD front view surface cut, with no high pressure air flow through the BSMs, $C_{p}$

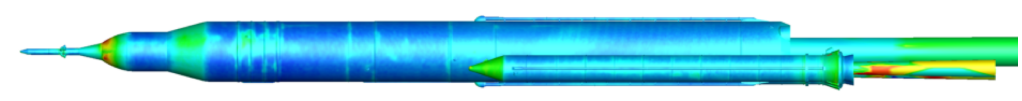

(c) PSP side view, with no high pressure air flow through the BSMs, $C_{p}$

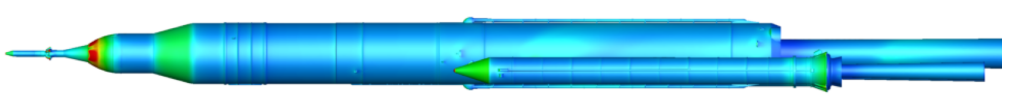

(d) CFD side view surface cut, with no high pressure air flow through the BSMs, $C_{p}$

Fig. 27 PSP image from tunnel testing versus a CFD $C_{p}$ solution at the same model location, with no high pressure air flow through the BSMs, Mach $=4.0$, and $\operatorname{Re}=1.25$ million/foot.

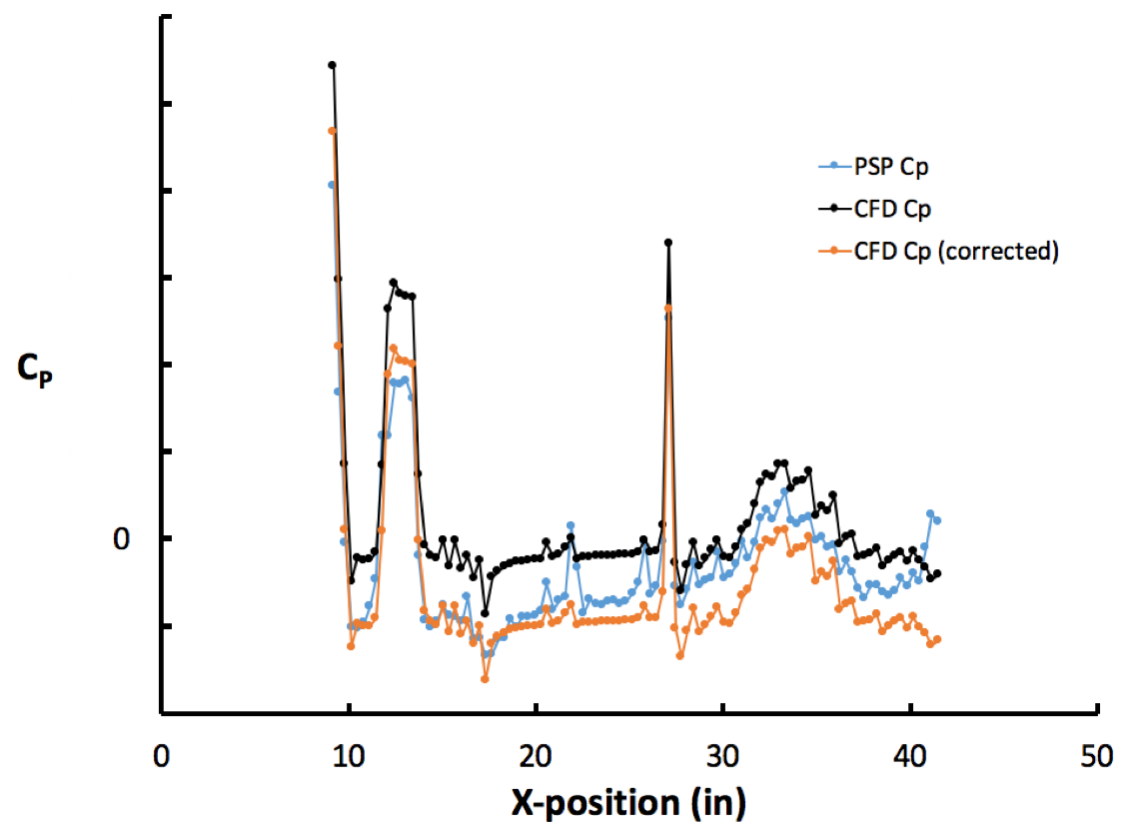

Fig. $28 \mathrm{X}$-position on the model (note that $\mathrm{X}=0$ is ahead on the LAS nose) vs. $C_{p}$, for PSP, CFD, and CFD corrected.

components and was used to fit and analyze all the corresponding PSP data. However, the PSP analysis program does not save this deformed grid, which accounts for the visual difference in the SRB positions between the PSP and CFD grids shown in Figure 29 The orientation seen in the CFD views is the correct visual orientation for how the PSP and CFD data were taken, but the PSP views do not show the correct orientation. There are some very obvious differences 
between the PSP data and the CFD comparison data when there is high pressure air flowing through the BSMs. The most obvious difference is that the PSP data is missing a lot of the high pressure region on the core that is predicted by the CFD (PSP is measuring a lower pressure). The high pressure air being run through the booster was heated to $150{ }^{\circ} \mathrm{F}$, however, when the high pressure air exits the booster it undergoes a rapid cooling, which manifests as a lower pressure in the PSP. Temperature effects are generally filtered out in PSP, but when two different air streams at different temperatures are interacting on the same model, the calibration can be affected. It is believed that the temperature effect from the air blowing out of the BSMs is causing the lower pressure to be seen in the PSP data on the core. It seems unlikely that having more pressure taps for reference would have helped since it appears the difference is caused by temperature and not pressure. Potentially a temperature compensating PSP would have been the solution and can be attempted on future tests with high pressure air usage.

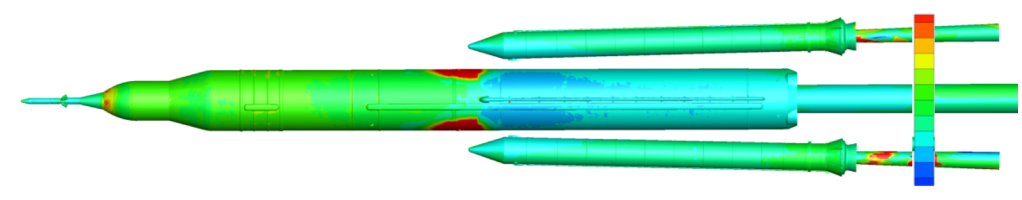

(a) PSP front view with high pressure air flow through the BSMs, $C_{p}$

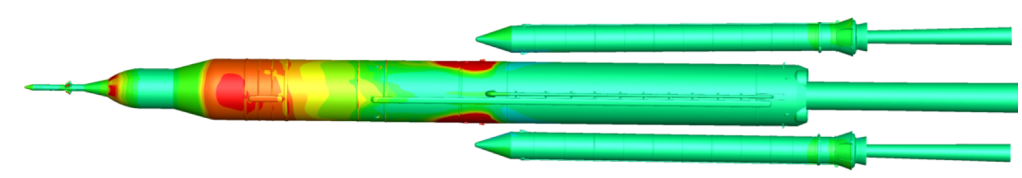

(b) CFD front view surface cut, with high pressure air flow through the BSMs, $C_{p}$

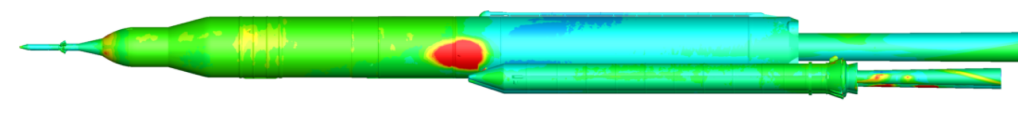

(c) PSP side view, with high pressure air flow through the BSMs, $C_{p}$

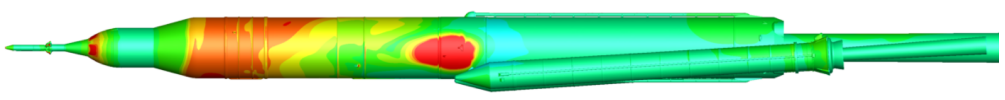

(d) CFD side view surface cut, with high pressure air flow through the BSMs, $C_{p}$

Fig. 29 PSP image from tunnel testing versus a CFD $C_{p}$ solution at the same model location, with high pressure air flow through the BSMs, Mach = 4.0, and $\operatorname{Re}=1.25$ million/foot.

\section{Conclusions}

A wind tunnel test was run in the NASA Langley UPWT simulating the separation of the two SRBs from the core stage of the SLS. The test was run on a 0.9\% scale model of the SLS Block 1B Cargo (27005) configuration and the SLS Block 1B Crew (28005) configuration at a Mach number of 4.0. High pressure air was used to simulate plumes from the booster separation motors located at the nose and aft skirt of the two boosters.

Along with the standard force and moment data, multiple surface and off-body measurement techniques were utilized to better understand the interaction between the SRBs and the core stage and to further validate CFD simulations. CFD simulations were run using FUN3D. CCDGV data were acquired to look at the vector velocity of the flow between the core stage and the boosters. Schlieren and flow visualization using a laser sheet were utilized to look at shock locations and interactions around the model. PSP data were acquired on the model body to look at $C_{p}$ on the surface of the vehicle. Comparisons between the CFD results and the surface and off-body measurements showed some good agreement, while some of the comparisons showed room for improvement in testing methods, especially when high pressure air was run through the BSMs to simulate jet flow.

While the CCDGV data and the CFD data did not fall right on top of each other, the trends did follow (for the no high pressure air flowing through the BSM cases). It can be noted that even the repeat CCDGV data does not fall on top of each other, because of the instability and dynamics in the area where the CCDGV was taken, and the CFD was run at 
full scale without walls. The CCDGV not matching with CFD when the high pressure air was being run through the BSMs will need to be studied more.

The PSP data with no high pressure air qualitatively followed the CFD predictions, with a slight offset in $C_{p}$. However, the PSP data showed much lower pressures on the core of the model when there was high pressure air flowing through the BSMs. This is believed to be due to temperature effects of the high pressure air blowing out of the BSMs. A change to a temperature compensating PSP for future testing will be considered.

It was believed that evaluating both surface and off-body measurement techniques during the SLS booster separation wind tunnel test would help validate CFD. Some of the visualization techniques helped with understanding of the flow around the model and matched well with CFD predictions. Other techniques, CCDGV and PSP, were found to have some issues with the high pressure airflow from the BSMs, and what was learned will help to improve future tests using these methods.

\section{Acknowledgments}

The authors would like to acknowledge the hard work performed by the NASA Langley Unitary Plan Wind Tunnel facility staff, engineers, and technicians throughout getting the tunnel recertified for this test, and throughout this test campaign. We would like to thank Sean Shea, Matthew Boyda, Gwi Bo Byun for their hard work on the CCDGV, and Bradley M. Wisser for his flow visulization work. We would also like to acknowledge the support by the Space Launch System program offices at the NASA Marshall Space Flight Center and at the NASA Langley Research Center, as well as the support from the SLS Aerodynamics Task Team.

\section{References}

[1] NASA, "NASA Space Launch System,”, 2018. URL http://www.nasa.gov/sls

[2] Wilcox, F., Pinier, J. T., Chan, D. T., and Crosby, W. A., "Space Launch System Booster Separation Aerodynamic Testing at the NASA Langley Unitary Plan Wind Tunnel," 54th AIAA Aerospace Sciences Meeting, 2016-0796, AIAA, San Diego, California, 2016.

[3] Rogers, S. E., Dalle, D. J., and Chan, W. M., "CFD Simulations of the Space Launch System Ascent Aerodynamics and Booster Separation," 53rd AIAA Aerospace Sciences Meeting, 2015-0778, AIAA, Kissimmee, Florida, 2015.

[4] Dalle, D. J., and Rogers, S. E., "Output-Based Adaptive Meshing Applied to Space Launch System Booster Separation Analysis," 33rd AIAA Applied Aerodynamics Conference, 2015-3152, AIAA, Dallas, TX, 2015.

[5] Blevins, J. A., Campbell, J. R., Bennett, D. W., Rausch, R., Gomez, R. J., and Kiris, C. C., "An Overview of the Characterization of the Space Launch System Aerodynamic Environments," 52nd Aerospace Sciences Meeting, 2014-1253, AIAA, National Harbor, Maryland, 2014.

[6] Chan, D. T., Dalle, D. J., Rogers, S. E., Pinier, J. T., Wilcox, F. J., and Gomez, R. J., "Space Launch System Booster Separation Aerodynamic Database Development and Uncertainty Quantification," 54th AIAA Aerospace Sciences Meeting, 2016-0798, AIAA, San Diego, California, 2016.

[7] Dalle, D. J., Rogers, S. E., Lee, H. C., and Chan, W. M., "Inviscid and Viscous CFD Analysis of Booster Separation for the Space Launch System Vehicle,” 54th AIAA Aerospace Sciences Meeting, 2016-0797, AIAA, San Diego, California, 2016.

[8] Pamadi, B. N., Pei, J., Gumbert, C. R., Green, L. L., Housman, J., Onufer, J., and Kiris, C. C., “Aerodynamic Modeling and Database Development of the Space Launch System Booster Separation," 53rd AIAA Aerospace Sciences Meeting, 2015-0779, AIAA, Kissimmee, Florida, 2015.

[9] Jackson, J., Charlie M., Corlett, W. A., and Monta, W. J., "Description and Calibration of the Langley Unitary Plan Wind Tunnel,” NASA Technical Paper 1905 1905, NASA, November 1981.

[10] Biedron, R. T., Calrson, J., Derlaga, J. M., Gnoffo, P. A., Hammond, D. P., Jones, W. T., Lee-Rausch, E. M., Nielson, E. J., Park, M. A., Rumsey, C. L., Thomas, J. L., and Wood, W. A., "FUN3D Manual: 13.1,” Tech. Rep. TM-2017-219580, NASA, 2017.

[11] Spalart, P. R., and Allmaras, S. R., "One-Equation Turbulence Model for Aerodynamic Flows," La Recherche Aerospatiale, Vol. 1, No. 1, 1994, pp. 5-21.

[12] “AFLR3 Unstructured Grid Generator," http://www.simcenter.msstate.edu/, 2018. SimSys Software, Version 15.8.11. 
[13] “ANSA Pre Processor,” https://www. beta-cae.com 2018. BETA CAE Systems, Version 16.0.4.

[14] Aftosmis, M., Berger, M., and Adomavicius, G., "A Parallel Multilevel Method for Adaptively Refined Cartesian Grids with Embedded Boundaries," 38th Aerospace Sciences Meeting, 2000. AIAA Paper 2000-0808.

[15] Erickson, G. E., "Overview of Supersonic Aerodynamics Measurement Techniques in the NASA Langley Unitary Plan Wind Tunnel,” NASA Technical Memo 2007-214894, NASA, August 2007.

[16] Lowe, K. T., Byun, G., Shea, S., Boyda, M., and Winski, C., "Three-Velocity-Component Cross-Correlation Doppler Global Velocimetry for the Space Launch System Booster Separation Test in the NASA Langley Unitary Plan Wind Tunnel," 57th AIAA Aerospace Sciences Meeting, 2019-2312, AIAA, San Diego, California, 2019.

[17] Danehy, P. M., Wisser, B. M., Fahringer, T. W., Winski, C. S., Falman, B. E., Shea, S., Boyda, M., and Lowe, K. T., "Laser Light Sheet Flow Visualization of the Space Launch System Booster Separation Test," 57th AIAA Aerospace Sciences Meeting, 2019-2313, AIAA, San Diego, California, 2019.

[18] Liu, T., and Sullivan, J. P., "Pressure and Temperature Sensitive Paints (Experimental Fluid Dynamics),” Springer-Verlag Berlin, Germany, 2004.

[19] Ruyten, W., Sellers, M., and Baker, W., "Spatially Nonuniform Self-Quenching of the Pressure-Sensitive Paint PtTFPP/FIB," 47th AIAA Aerospace Sciences Meeting, 2009-1660, AIAA, Orlando, Florida, 2009.

[20] Sellers, M., "Demonstration of a Temperature-Compensated Pressure Sensitive Paint on the Orion Launch Abort Vehicle (Invited)," 29th AIAA Applied Aerodynamics Conference, 2011-3166, AIAA, Honolulu, Hawaii, 2011.

[21] FARO, "FARO Arm,", 2018. URL https://www . faro.com/products/factory-metrology/faroarm/

[22] Sellers, M. E., "Pressure-Sensitive Paint Data on the Facility Aerodynamics Validation and Research (FAVOR) Model at AEDC," NASA Technical Report AEDC-TR-09-F-34, NASA, December 2009. 